\title{
On the streamwise evolution of turbulent boundary layers in arbitrary pressure gradients
}

\author{
By A. E. PERRY ${ }^{1} \dagger$, IVAN MARUSIC ${ }^{2}$ AND M. B. JONES \\ ${ }^{1}$ Department of Mechanical and Manufacturing Engineering, University of Melbourne, Parkville, \\ Victoria 3052, Australia \\ ${ }^{2}$ Department of Aerospace Engineering and Mechanics, University of Minnesota, Minneapolis, \\ MN 55455 USA
}

(Received 26 February 1999 and in revised form 12 December 2001)

\begin{abstract}
A new approach to the classic closure problem for turbulent boundary layers is presented. This involves, first, using the well-known mean-flow scaling laws such as the log law of the wall and the law of the wake of Coles (1956) together with the mean continuity and the mean momentum differential and integral equations. The important parameters governing the flow in the general non-equilibrium case are identified and are used for establishing a framework for closure. Initially closure is achieved here empirically and the potential for achieving closure in the future using the wall-wake attached eddy model of Perry \& Marusic (1995) is outlined. Comparisons are made with experiments covering adverse-pressure-gradient flows in relaxing and developing states and flows approaching equilibrium sink flow. Mean velocity profiles, total shear stress and Reynolds stress profiles can be computed for different streamwise stations, given an initial upstream mean velocity profile and the streamwise variation of free-stream velocity. The attached eddy model of Perry \& Marusic (1995) can then be utilized, with some refinement, to compute the remaining unknown quantities such as Reynolds normal stresses and associated spectra and cross-power spectra in the fully turbulent part of the flow.
\end{abstract}

\section{Introduction}

The motivation for this work is to develop a framework for incorporating coherent structure concepts into the classic closure problem of turbulent boundary layer streamwise evolution. The closure scheme makes as much use as possible of the classical similarity laws which have won wide support, namely, the logarithmic law of the wall and the law of the wake of Coles (1956). When used in conjunction with the usual boundary layer mean momentum differential equation and the mean continuity equation, expressions for the total shear stress distribution can be rigorously derived in the manner of Perry, Marusic \& Li (1994). From this, four non-dimensional parameters emerge which describe the state of the layer. It is assumed that there exists a universal relationship among these four parameters. This relationship must be determined experimentally and existing data are sparse. An interpolation and extrapolation scheme is devised so that flow cases outside and between regions already observed can be computed. The extension of the Townsend (1976) attached eddy hypothesis by Perry \& Marusic (1995) and Marusic \& Perry (1995) aids in

$\dagger$ Sadly, Professor Perry died on 3 January 2001 during the preparation of this paper. 
this interpolation and extrapolation. The application of this extended attached eddy hypothesis is yet to be completed, but this initial attempt forms a backbone for the empirical formulation.

With the aid of the momentum integral equation, a pair of coupled nonlinear firstorder ODEs emerges which can be solved so that the streamwise evolution of the layer can be computed. All that is needed is the specification of an initial velocity profile and the streamwise distribution of free-stream velocity. One interesting outcome is that a nonlinear dynamical system approach to the evolution of turbulent boundary layers emerges here which is similar to the famous idea first expressed by Clauser (1956) with his black box analogy where the independent time variable in dynamical systems is replaced by a variable related to the streamwise coordinate $x$. The resulting ODEs are autonomous or non-autonomous depending on the external flow geometry. If the equations are autonomous, phase-plane trajectories can be mapped out for different initial conditions and these trajectories, in some cases, can converge to critical points or equilibrium points which correspond to the precise equilibrium flow discussed by Rotta (1962).

From an extension of the Townsend (1976) attached eddy hypothesis Perry \& Marusic (1995) and Marusic \& Perry (1995) showed that given the streamwise development of the mean-velocity field and Reynolds shear stress field, the Reynolds normal stresses and associated spectra can be evaluated. The present approach allows the streamwise development of the mean-velocity field to be computed. Although the proposed approach is restricted, further work will surely generalize it. The evolution calculations are currently restricted to two-dimensional flow on a flat surface with an imposed streamwise pressure gradient. Flow separation cannot yet be handled. However, some preliminary unpublished work by the authors shows that surface roughness might perhaps be incorporated quite easily. Recently, lateral divergence has been included in the shear stress formulation giving the potential for extensions. Over certain regions of the various parameters the closure is, in principle, complete. All that is required is the specification of an initial upstream mean velocity profile and the streamwise variation of the free-stream velocity. From this the streamwise evolution of all mean velocity profiles and total shear stress profiles can be computed.

It should be emphasized that the present aim of this work is not to produce a viable code for general engineering computations but to develop a framework for the study of the dynamics of turbulent boundary layer evolution for simple cases. At the moment the attached eddy hypothesis is a kinematic description and this framework will aid in putting some dynamics into the physical modelling. Finally, if the assumptions involved are verified with further data, the closure scheme could serve as a tool for producing accurate benchmark computations of simple cases for the testing of more complex and speculative methods.

\section{Shear stress profiles}

As stated earlier, Perry et al. (1994) derived a general analytical expression for the shear stress profile for a flat-plate turbulent boundary layer evolving in a streamwise pressure gradient. This is summarized here. The usual boundary layer mean momentum equation where streamwise gradients of normal stresses are negligible is given by (e.g. see Rotta 1962)

$$
U \frac{\partial U}{\partial x}+W \frac{\partial U}{\partial z}=-\frac{1}{\rho} \frac{\mathrm{d} p_{1}}{\mathrm{~d} x}+\frac{1}{\rho} \frac{\partial \tau}{\partial z}
$$


where

$$
\frac{\tau}{\rho}=-\overline{u_{1} u_{3}}+v \frac{\partial U}{\partial z} .
$$

Here $U$ is the mean streamwise component of velocity, $W$ is the mean normal to the wall velocity, $x$ is the streamwise coordinate, $z$ is normal to the wall, $u_{1}$ and $u_{3}$ are the streamwise and wall-normal fluctuating components of velocity, an overbar denotes a temporal mean, $p_{1}$ is the free-stream static pressure and $v$ is the kinematic viscosity. The mean continuity equation is given by

$$
\frac{\partial U}{\partial x}+\frac{\partial W}{\partial z}=0
$$

and if $U_{1}$ is the free-stream velocity, then

$$
-\frac{1}{\rho} \frac{\mathrm{d} p_{1}}{\mathrm{~d} x}=U_{1} \frac{\mathrm{d} U_{1}}{\mathrm{~d} x} .
$$

It is assumed that the mean velocity profile can be described by the Coles (1956) log law of the wall and law of the wake formulation, i.e.

$$
\frac{U}{U_{\tau}}=\frac{1}{\kappa} \log \left[\frac{z U_{\tau}}{v}\right]+A+\frac{\Pi}{\kappa} W_{c}[\eta, \Pi] .
$$

Here $U_{\tau}$ is the local friction velocity, $\Pi$ is Coles' wake factor, $\eta=z / \delta_{c}$ where $\delta_{c}$ is the boundary layer thickness, and $\kappa$ and $A$ are universal constants. $W_{c}$ is the universal wake function and many functional forms have been proposed. The logarithmic law of the wall is used and for the purpose of computing the total shear stress and overall momentum balance, it is assumed valid right to the wall. For practical ranges of Reynolds numbers this approximation is justified as was shown by Jones, Marusic \& Perry (2001). Equation (2.5) can be expressed in velocity defect form as

$$
\begin{aligned}
\frac{U_{1}-U}{U_{\tau}} & =-\ln [\eta]+\frac{\Pi}{\kappa} W_{c}[1, \Pi]-\frac{\Pi}{\kappa} W_{c}[\eta, \Pi] \\
& =f[\eta, \Pi]
\end{aligned}
$$

and so

$$
U=U_{1}-U_{\tau} f[\eta, \Pi] .
$$

By substituting (2.7) into the continuity equation (2.3) an expression for the normal velocity $W$ is obtained in terms of the other mean flow variables and their streamwise derivatives. The expressions for $U$ and $W$ along with their appropriate derivatives can then be substituted into (2.1). This is then integrated with respect to $z$ and using (2.4) as a boundary condition an expression for the total shear stress is obtained and is given as (A 1) in Appendix A. Further details are given in that Appendix.

Four non-dimensional parameters emerge which describe the state of the layer: they are

$$
\Pi, \quad S=\frac{U_{1}}{U_{\tau}}, \quad \beta=\frac{\delta^{*}}{\tau_{0}} \frac{\mathrm{d} p}{\mathrm{~d} x}, \quad \zeta=S \delta_{c} \frac{\mathrm{d} \Pi}{\mathrm{d} x} .
$$

Here $S$ is a skin friction parameter where $U_{1}$ is the local free-stream velocity, $\beta$ is the Clauser $(1954,1956)$ pressure gradient parameter where $\delta^{*}$ is the displacement thickness, $\tau_{0}$ is wall shear stress, $p$ is the free-stream static pressure, $x$ is streamwise distance and $\zeta$ is a non-equilibrium parameter. The total shear stress is given by

$$
\frac{\tau}{\tau_{0}}=f_{1}[\eta, \Pi, S]+g_{1}[\eta, \Pi, S] \zeta+g_{2}[\eta, \Pi, S] \beta .
$$


Here $f_{1}, g_{1}$ and $g_{2}$ are known universal analytical functions and their precise form will depend on the functional form chosen for $W_{c}$. Their formulations are long, each requiring several pages and can be found using Mathematica or Maple. Their structure is discussed in Perry \& Marusic (1995). One important property of $f_{1}, g_{1}$ and $g_{2}$ is that they become independent of $S$ for $S$ sufficiently large. It should be noted that $g_{1}$ is negative and $f_{1}$ and $g_{2}$ are positive quantities. Thus a positive $\zeta$ causes a decrease in shear stress. Throughout this paper, the streamwise gradient of normal stresses has been neglected and this was found to be valid for all experimental data considered here.

\section{Closure formulation for approximate-equilibrium and quasi- equilibrium flow}

Perry et al. (1994) used the shear stress information to develop a closure scheme for calculating the streamwise evolution of a turbulent boundary layer developing in an arbitrary pressure gradient. The cases they considered were not completely arbitrary and were confined to flows which were either in approximate equilibrium or quasi-equilibrium. In approximate equilibrium flows, $\Pi$ is assumed to be constant and in quasi-equilibrium flows, $\Pi$ is allowed to vary with $x$ but this variation is sufficiently slow to keep the effects of the parameter $\zeta$ small. From (2.9) this would occur if

$$
\left|\frac{g_{1}[\eta, \Pi, S] \zeta}{\tau / \tau_{0}}\right| \ll 1
$$

In both cases the shear stress profiles are characterized only by $\Pi$ (approximately). In this early work it was not yet clear how $\zeta$ could be incorporated. In this paper we will consider the general non-equilibrium case where finite $\zeta$ values are taken into account. However let us briefly review this earlier work.

If $\Pi$ is invariant with $x$, then we have a self-similar velocity defect distribution and let us assume that this implies a self-similar shear stress distribution. We know that this is not possible precisely since Rotta (1962) has shown that for smooth wall flows precise equilibrium is possible only in sink flow. However, perhaps we can find conditions with the aid of (2.9) which will give approximate self-similarity of the shear stress distributions for a fixed $\Pi$. We will refer to this condition as 'approximate equilibrium'. Let us force all shear stress profiles for a fixed $\Pi$ to match at $\eta=m$ for all $S$. Then (2.9) gives

$$
\frac{\tau}{\tau_{0}}[m, \Pi]=\mathscr{L}[m, \Pi]=f_{1}[m, \Pi, S]+g_{2}[m, \Pi, S] \beta .
$$

Note that since $\Pi$ is invariant with $x, \zeta=0$. It turns out that with $m=0.4$, quite reasonable self-similarity of shear stress profiles is possible for each $\Pi$ for the whole range of $\Pi$ values over the full range of $S$. See Perry (1992) for details. From (3.2) a relationship among $\Pi, \beta$ and $S$ can be constructed with the aid of experimental data. Suppose that for a fixed $x, \Pi, S$ and $\beta$ are known from experiment. Hence $\mathscr{L}[m, \Pi]$ is known and if we keep $\Pi$ fixed and vary $S$ we can see how $\beta$ must vary to ensure approximate self-similarity of the shear stress profiles. Now it can be seen from the structure of (2.9) detailed in (A 8) that as $S \rightarrow \infty,(2.9)$ becomes invariant with $S$ and so $\beta$ approaches an asymptotic value of $\beta_{a}$ for fixed $\Pi$. If this can be carried out with a sufficient number of experimental flow cases, we can determine

$$
\beta_{a}=\beta_{a}[\Pi] .
$$


With the aid of (3.2) and (3.3) we can map out the relationship

$$
C[\Pi, \beta, S]=0,
$$

which is assumed universal for all approximate-equilibrium flows. If we take this further and allow $\Pi$ to vary slowly with $x$ but with $\zeta$ sufficiently small to ignore, then we have what we will call 'quasi-equilibrium' flow and (3.4) is assumed to be still valid.

Clauser $(1954,1956)$ originally postulated that (approximate) equilibrium layers could be achieved by keeping $\beta$ constant (i.e. invariant with streamwise distance $x$ and hence $S$ ) but Perry (1992) showed this gives poor self-similarity of the shear stress profiles for the whole range of possible $S$ and, as was just shown, $\beta$ must vary with $S$. Other criteria postulated in the past were also tested and Perry (1992) showed that when the momentum integral equation is used all theories and conditions tested for approximate equilibrium yielded $S \rightarrow \infty$ as the streamwise Reynolds number $R_{x}=\left(x U_{0} / v\right) \rightarrow \infty$, where $U_{0}$ is a reference free-stream velocity at an arbitrarily chosen value of $x$ and $v$ is the kinematic viscosity. It turns out that as $S \rightarrow \infty$ we achieve precise equilibrium asymptotically, i.e. perfect self-similar shear stress profiles.

At infinite $S, C$ is no longer a function of $S$. If this procedure is repeated for different values of $\Pi$, a one-to-one relationship between $\beta_{a}$ and $\Pi$ can be found empirically. There have been various curve-fits for $\beta$ and $\Pi$ in the literature (although in past work $S$ was assumed finite and the effect of the variable $S$ was ignored). White (1974) proposed

$$
\Pi=0.8\left(\beta_{a}+0.5\right)^{0.75} .
$$

Formulations which assume that both the velocity defect and shear stress distributions are controlled by the one parameter $\Pi$ automatically imply that we have a universal relation for eddy viscosity $\epsilon$ of the form

$$
\epsilon /\left(\delta_{c} U_{\tau}\right)=\phi[\eta, \Pi] .
$$

This does not necessarily mean that there is a gradient diffusion mechanism at work but by default (3.6) works for the quasi-equilibrium cases. Unfortunately, such formulations are known to break down in non-equilibrium flows. Figure 1, which is taken from Marusic \& Perry (1995), shows clearly how flows with the same $\Pi$ value can have very different shear stress distributions. In the last part of his paper, Clauser (1956) effectively developed the concept of the universal eddy viscosity distribution given by (3.6) for approximate-equilibrium layers. This was taken up by many workers who applied it to non-equilibrium layers.

\section{New formulation for general flows}

The restricted formulation of Perry et al. (1994) will now be extended with the effect of the parameter $\zeta$ included so that the non-equilibrium flow problem can be solved. Therefore, the function (3.4) needs to be replaced by

$$
\mathscr{F}[\Pi, S, \beta, \zeta]=0
$$

and so we have to work in a higher dimensional space than did Perry et al. (1994). It is assumed that no further parameters are involved in (4.1) and $\mathscr{F}$ is universal. Hence in order to describe the state of the layer, we require three of the four variables in the above expression. The mapping out of equation (4.1) from experimental data would be extremely difficult because of the sparseness of the data. In what follows, 


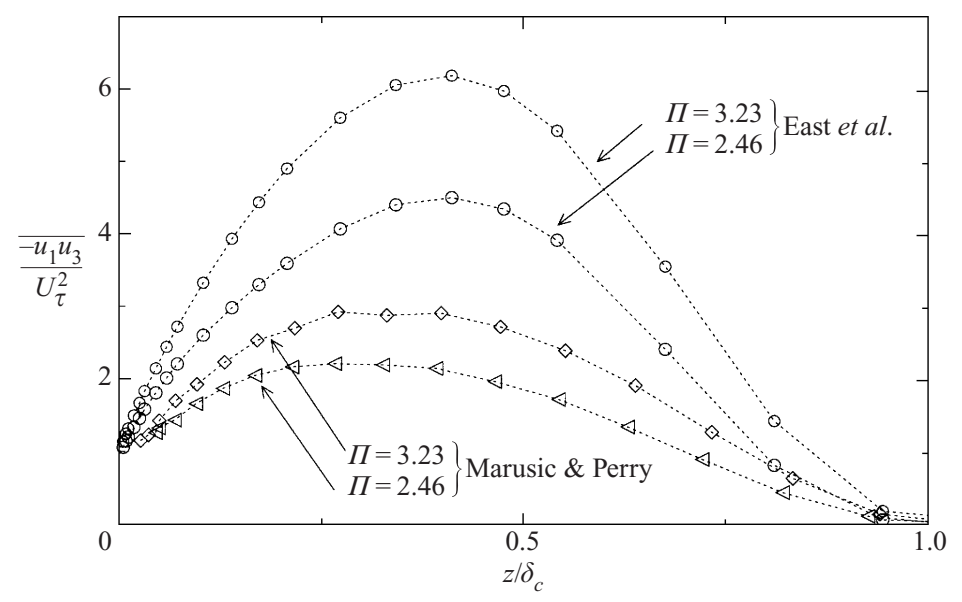

FIGURE 1. Comparison of non-equilibrium data of Marusic \& Perry (1995) where $\Pi=2.46$ and 3.23 (10APG) with interpolated data for the same values of $\Pi$ for the equilibrium flow of East, Sawyer \& Nash (1979). Lewkowicz's (1982) profile formulation used for $\Pi$ (see $\S 4$ ).

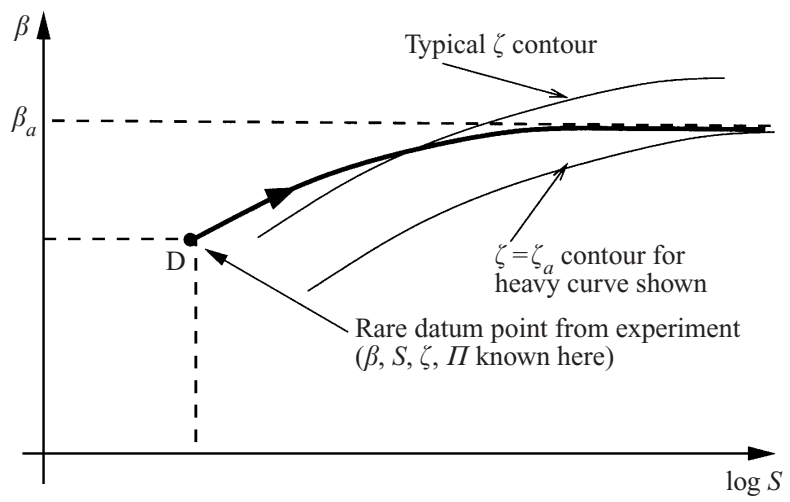

Figure 2 . The $(S, \beta)$-plane at fixed $\Pi$. Heavy solid line indicates the trajectory of the least-squares error in shear stress profile matching with known profile at D.

a mathematical framework for interpolation and extrapolation with sparse data is developed. In analogy with $C$ in (3.4) $\mathscr{F}$ is independent of $S$ for $S \rightarrow \infty$ and $\beta$ and $\zeta$ reach asymptotic values of $\beta_{a}$ and $\zeta_{a}$ for a fixed $\Pi$.

From figure 1 it is obvious that the shear stress distribution needs at least two parameters to describe it and we will assume that

$$
\frac{\tau}{\tau_{0}}=f\left[\eta, \Pi, \beta_{a}\right]
$$

It will be shown a little later that (4.2) is valid for infinite $S$. It is hypothesized that this two-parameter relation is also valid and unchanged for finite $S$. For quasi-equilibrium flows Perry et al. (1994) relied on a one-parameter family to describe the shear stress, i.e. $\tau / \tau_{0}=f[\eta, \Pi]$ where $f$ is assumed to be universal.

If the two-parameter property of (4.2) is used in conjunction with (2.9), some information can be obtained regarding (4.1) as follows. Consider the $(S, \beta)$-plane at a fixed $\Pi$ as shown in figure 2. If such a plane contains an experimental datum point $\mathrm{D}$, then $S, \Pi, \beta$ and $\zeta$ are known for point $\mathrm{D}$ from experiment and so also is 
$\tau / \tau_{0}$ versus $\eta$ from (2.9). From point $\mathrm{D}$ trace out on the $(S, \beta)$-plane a curve along which points have the same distribution of $\tau / \tau_{0}$ versus $\eta$ as occurs at point $\mathrm{D}$. This is done by optimizing $\beta$ and $\zeta$ for different $S$ such that the best matching occurs with the distribution at $\mathrm{D}$. The matching may not be precise but hopefully will be close enough for practical purposes. Therefore at each point along the heavy curve shown in figure 2 we will know $\beta$ and $\zeta$.

By taking $S \rightarrow \infty$ we obtain asymptotic values of $\beta_{a}$ and $\zeta_{a}$ as shown in figure 2 . (Going to $S=\infty$ is simply a convenient curve-fitting procedure and could never be approached experimentally.) This process of keeping the profile distribution fixed will be referred to as 'profile matching' and the details of the method used here are given in the following section. If this process is repeated often enough for different $\Pi$ then we obtain a $\Pi, \beta_{a}$ diagram with distributions of extrapolated data points corresponding to different values of $\zeta_{a}$. By a surface fit to $\zeta_{a}$ on the $\left(\Pi, \beta_{a}\right)$-plane, contours of $\zeta_{a}$ can be mapped out and we thus have a known universal function $\psi$ :

$$
\psi\left[\Pi, \beta_{a}, \zeta_{a}\right]=0 .
$$

Closure rests completely on (4.3) and this relation involves only three variables. By shear stress profile matching we can then map out isosurfaces of $\zeta$ in $\Pi, \beta, S$ space and thus (4.1) is known (see later). Let us show that (4.2) is valid for infinite $S$. From (2.9) as $S \rightarrow \infty$ the only parameters which control $\tau / \tau_{0}$ are $\Pi, \beta_{a}$ and $\zeta_{a}$, and from (4.1) we have (4.3) (i.e. $\psi\left[\Pi, \beta_{a}, \zeta_{a}\right]=0$ ). It is seen that for a fixed $\Pi$ specification of $\beta_{a}$ also specifies $\zeta_{a}$ and no further parameters are required. As stated earlier, this two-parameter property is assumed valid at finite $S$ which effectively means that shear stress profile matching is possible at all $\mathrm{S}$ for a fixed $\Pi$.

\subsection{Profile matching}

The shear stress profile matching technique used here involves using a least-squares error criterion to find optimum $\beta$ and $\zeta$ for a fixed $\Pi$ and $S$. We find

$$
\left.\begin{array}{l}
\frac{\partial}{\partial \beta}\left\{\int_{0}^{1}\left[\left(\frac{\tau}{\tau_{0}}\right)-\left(\frac{\tau}{\tau_{0}}\right)_{D}\right]^{2} \mathrm{~d} \eta\right\}=0, \\
\frac{\partial}{\partial \zeta}\left\{\int_{0}^{1}\left[\left(\frac{\tau}{\tau_{0}}\right)-\left(\frac{\tau}{\tau_{0}}\right)_{D}\right]^{2} \mathrm{~d} \eta\right\}=0,
\end{array}\right\}
$$

where $\tau / \tau_{0}$ is the shear stress distribution at any point on the $(\beta, S)$-plane for fixed $\Pi$ and $\left(\tau / \tau_{0}\right)_{D}$ is the shear stress distribution at a known datum point, e.g. point $\mathrm{D}$ in figure 2. For a well-defined matching trajectory the solid line in figure 2 would correspond to a deep valley on a contour plot of least-square error. Taking (4.4) to $S \rightarrow \infty$ means that we use $\partial / \partial \beta_{a}$ and $\partial / \partial \zeta_{a}$ as the derivatives and we can show generally that

$$
\left.\begin{array}{l}
A_{1}[\Pi, S]+B_{1}[\Pi, S] \zeta+C_{1}[\Pi, S] \beta=D_{1}[\Pi] \zeta_{a}+E_{1}[\Pi] \beta_{a}, \\
A_{2}[\Pi, S]+B_{2}[\Pi, S] \zeta+C_{2}[\Pi, S] \beta=D_{2}[\Pi] \zeta_{a}+E_{2}[\Pi] \beta_{a},
\end{array}\right\}
$$

where $A_{1}, A_{2}, B_{1}, B_{2}$ etc. are all known analytical functions. Their functional forms will depend on the type of wall-wake formulation chosen. Several forms have been proposed and in the Perry et al. (1994) study the formulation due to Lewkowicz 
(1982) was used. Here we will use the recently proposed formulation of Jones et al. (2001)

$$
\frac{U}{U_{\tau}}=\underbrace{\overbrace{\frac{1}{\kappa} \ln \left[\frac{z U_{\tau}}{v}\right]+A}^{\text {Log-law of the wall }}-\frac{1}{3 \kappa} \eta^{3}}_{\text {Pure wall flow }}+\overbrace{\frac{\Pi}{\kappa} 2 \eta^{2}(3-2 \eta)}^{\text {Pure wake component }}
$$

where $\kappa=0.41$ is the Kármán constant and $A$ is the universal smooth-wall constant taken here to be 5.0. This formulation was found to work particularly well in describing the 'pure-wall' component of the flow as found in sink flows. Note that with this type of functional form the maximum deviation from the log law is not the classic $2 \Pi_{c} / \kappa$ but will be a nonlinear function of $\Pi$-see Jones et al. (2001). Here $\Pi_{c}$ is the classical value for the Coles wake function and is related to $\Pi$ by

$$
\Pi_{c}=\frac{144 \Pi^{3}}{(12 \Pi+1)^{2}} \text {. }
$$

It should be noted that once $U_{\tau}$ has been found (e.g. by the Clauser chart method) (4.6) can be used to devise several rigorous and systematic methods for determining $\Pi$ and $\delta_{c}$, see Appendix $\mathrm{C}$ for one example.

Figure 3(a) shows a comparison of how well the resulting profile matching works for fixed $\Pi$ with $S$ varying from the values obtained from experiment up to $S=10^{8}$ for the data of Marusic \& Perry (1995). Each $\Pi$ case appears as a single curve but is actually five curves superimposed for different values of $S$ for optimum $\beta$ and $\zeta$. This is a great improvement over the matching technique of Perry et al. (1994) where shear stresses were crudely forced to match at $\eta=0.4$ as given in $\S 3$. Figures $3(b)$ and $3(c)$ show similar matching calculations for the flows of Bradshaw \& Ferriss (1965) and Samuel \& Joubert (1974). These figures show that (4.2) is possible for all $S$, at least on theoretical grounds.

\subsection{New evolution equations}

The equations which govern the streamwise evolution of a turbulent boundary layer can be found after considerable algebra by using the momentum integral equation, the log law of the wall and law of the wake together the definitions for $\beta$ and $\zeta$. Some details are given in Appendix B. A coupled set of ODEs results:

$$
\frac{\mathrm{d} S}{\mathrm{~d} R_{x}}=\frac{\chi\left[R_{x}, R_{L}\right] R[S, \Pi, \zeta, \beta]}{S E[\Pi] \exp [\kappa S]},
$$

and

$$
\frac{\mathrm{d} \Pi}{\mathrm{d} R_{x}}=\frac{\zeta \chi\left[R_{x}, R_{L}\right]}{S^{2} E[\Pi] \exp [\kappa S]} .
$$

Two auxiliary equations are needed to solve for the integration. The first is (4.1), i.e.

$$
\mathscr{F}[\Pi, S, \beta, \zeta]=0 .
$$

This relation is generated from (4.3) and (4.5). The second auxiliary equation is

$$
S^{2} E[\Pi] \exp [\kappa S] \frac{1}{\chi^{2}} \frac{\mathrm{d} \chi}{\mathrm{d} R_{x}}=-\frac{\beta}{C_{1}[\Pi]} .
$$

Equation (4.8) comes from the momentum integral equation and the log law of the wall and law of the wake. Equation (4.9) comes from the log law of the wall and law 


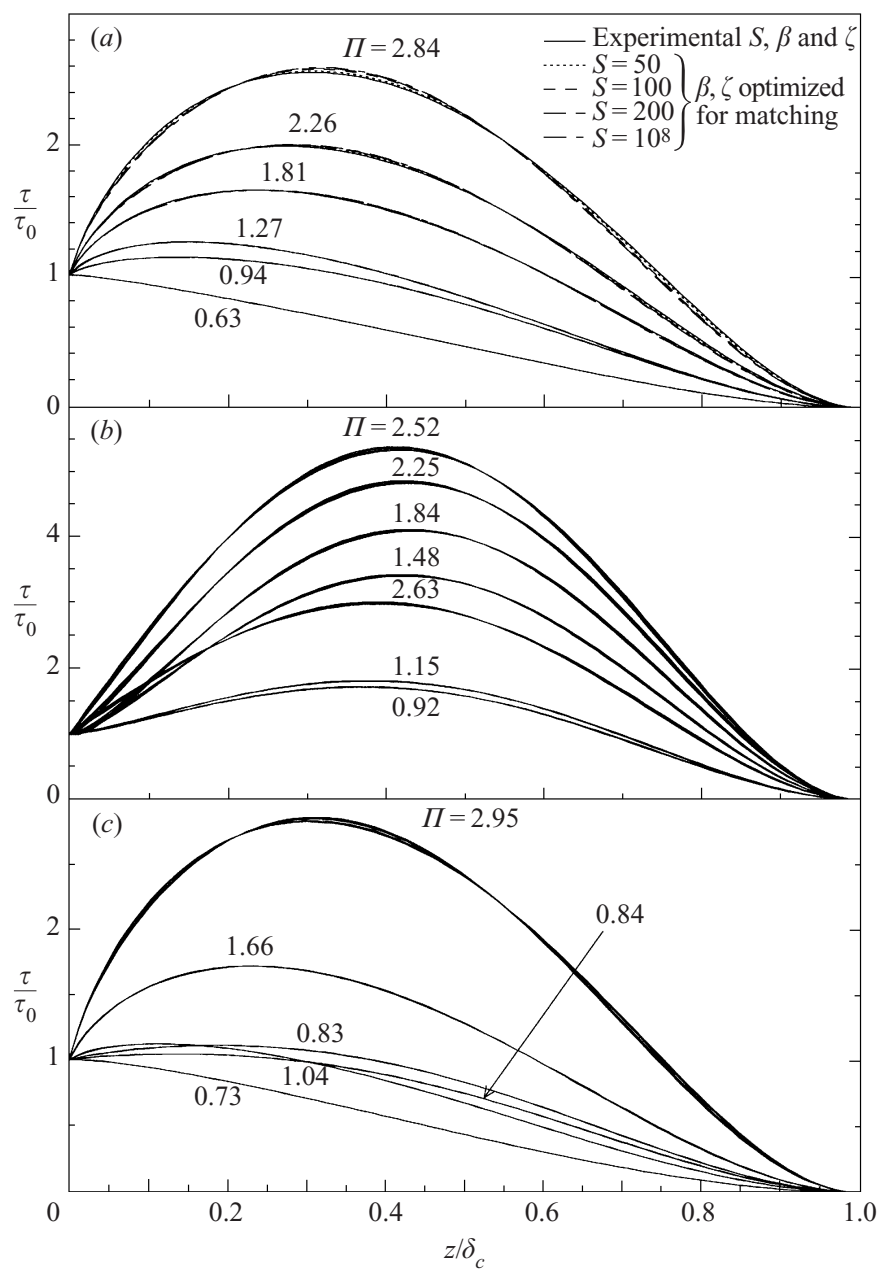

FIGURE 3. Matched shear stress profiles using (2.9) and (4.4) for fixed experimental $\Pi$ with different values of $S$. Five curves are present for each value of $\Pi$. The heavy line was computed using the experimental values of $S, \beta$ and $\zeta$ from (a) Marusic \& Perry (1995) 30 APG, $(b)$ Bradshaw \& Ferriss (1965) and (c) Samuel \& Joubert (1974).

of the wake and from the definition of $\zeta$. Equation (4.10) comes from from the log law of the wall and law of the wake and the definition of $\beta$. The functions appearing in (4.8) and (4.9) are defined as follows:

$$
\begin{aligned}
& R[S, \Pi, \beta, \zeta]= \\
& \quad \frac{S+\beta\left(2 S-C_{2}[\Pi] / C_{1}[\Pi]\right)+\zeta\left(C_{1}[\Pi] S N-C_{2}[\Pi] N-S \frac{\mathrm{d} C_{1}[\Pi]}{\mathrm{d} \Pi}+\frac{\mathrm{d} C_{2}[\Pi]}{\mathrm{d} \Pi}\right)}{\kappa C_{1}[\Pi] S^{2}-\kappa C_{2}[\Pi] S+C_{2}[\Pi]}
\end{aligned}
$$

where

and from (4.6)

$$
N=W_{c}[1, \Pi]+\Pi \frac{\mathrm{d} W_{c}[1, \Pi]}{\mathrm{d} \Pi}
$$

$$
W_{c}[\eta, \Pi]=-\frac{1}{3 \Pi} \eta^{3}+2 \eta^{2}(3-2 \eta)
$$


from which it follows that $N=2$. Also

$$
\begin{aligned}
& C_{1}[\Pi]=\int_{0}^{1} \frac{U_{1}-U}{U_{\tau}} \mathrm{d} \eta=(\Pi+3 / 4) / \kappa, \\
& C_{2}[\Pi]=\int_{0}^{1}\left(\frac{U_{1}-U}{U_{\tau}}\right)^{2} \mathrm{~d} \eta=\left(1248 \Pi^{2}+2140 \Pi+1215\right) /\left(840 \kappa^{2}\right)
\end{aligned}
$$

and

$$
\begin{gathered}
E[\Pi]=\exp \left[-\kappa\left(A+\frac{\Pi}{\kappa} W_{c}[1, \Pi]\right)\right], \\
R_{x}=\frac{x U_{0}}{v}, \quad R_{L}=\frac{L U_{0}}{v},
\end{gathered}
$$

where $U_{0}$ is the value of the free-stream velocity at some initial point $R_{x}=0, U_{1}$ is the free-stream velocity at some general value of $x$ or $R_{x}$ and $L$ is a characteristic length scale of the $U_{1}$ distribution. $R_{L}$ is an overall Reynolds number of the apparatus and $\chi\left[R_{x}, R_{L}\right]=U_{1} / U_{0}$; usually $\chi=\chi\left[R_{x} / R_{L}\right]$, i.e. $\chi=\chi[x / L]$. This latter form would occur if the free-stream velocity distribution was independent of Reynolds number, i.e. when boundary layer displacement thickness effects can be ignored and no other viscid-inviscid interactions are occurring. All of the above equations can be reduced to two coupled first-order nonlinear ODEs of the form

$$
\begin{aligned}
\frac{\mathrm{d} S}{\mathrm{~d} R_{x}} & =\phi_{1}\left[\Pi, S, R_{x}, R_{L}\right], \\
\frac{\mathrm{d} \Pi}{\mathrm{d} R_{x}} & =\phi_{2}\left[\Pi, S, R_{x}, R_{L}\right] .
\end{aligned}
$$

\section{Application of the extended attached eddy hypothesis}

The preceding section describes the theory required to compute the evolution of the mean flow field of a turbulent boundary layer. In principle, this could be done using empirical information (4.3) and hence (4.1). Here we explore the viability of employing the attached eddy hypothesis to assist in finding a description for the formulation $\psi\left[\Pi, \beta_{a}, \zeta_{a}\right]=0$.

Perry et al. (1994) attempted to apply the attached eddy hypothesis for determining $\beta_{a e}=\beta_{a e}[\Pi]$ with some limited success. Here $\beta_{a e}=\beta_{a}$ for $\zeta_{a}=0$. They assumed that all eddies were of type A as shown in their figure 2.9(a) whose vortex cores extended to the wall. They used two convolution integrals first derived by Perry, Li \& Marusic (1991):

$$
\begin{aligned}
\frac{\mathrm{d} U_{D}{ }^{*}}{\mathrm{~d} \lambda_{E}} & =\int_{-\infty}^{\infty} h[\lambda] \mathrm{e}^{-\lambda} T\left[\lambda-\lambda_{E}\right] w\left[\lambda-\lambda_{E}\right] \mathrm{d} \lambda \\
\frac{\overline{u_{i} u_{j}}}{U_{\tau}^{2}} & =\int_{-\infty}^{\infty} J_{i j}[\lambda] T^{2}\left[\lambda-\lambda_{E}\right] w\left[\lambda-\lambda_{E}\right] \mathrm{d} \lambda .
\end{aligned}
$$

Here $\lambda_{E}=\ln \left(\delta_{c} / z\right)$ and $\lambda=\ln (\delta / z)$ where $\delta$ is the eddy length scale. $T$ is a weighting function dependent on the characteristic velocity scale of an eddy of length scale $\delta$ and $w$ is a weighting factor dependent on the wall-surface-area population density of eddies of length scale $\delta$. Also, $h[\lambda]$ is a vorticity function dependent on eddy shape and $J_{i j}$ is the Townsend (1976) eddy intensity function also dependent on eddy shape. All eddies are assumed to be geometrically similar. In Perry et al. (1994) by 

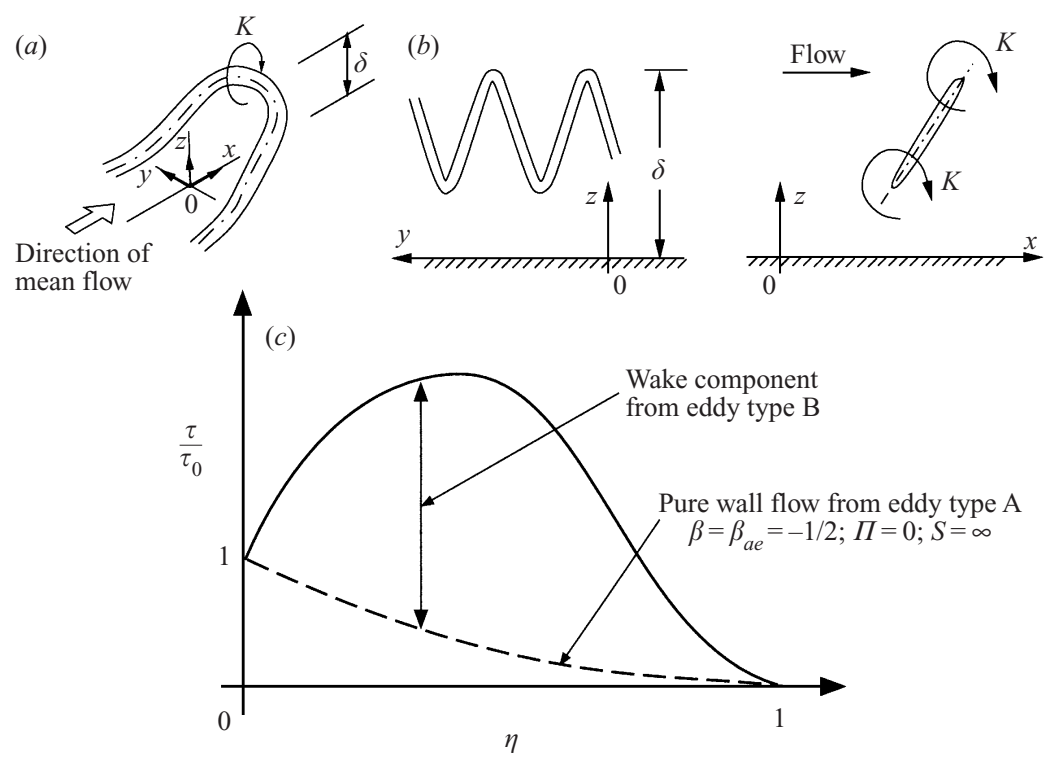

FIGURE 4. (a) Sketch of a representative wall eddy, i.e. type A, $(b)$ sketch of a representative wake eddy, i.e. type B and $(c)$ type $\mathrm{A}$ and type $\mathrm{B}$ eddy contributions to the total shear stress. $(a, b)$ After Perry \& Marusic (1995) and Chong et al. (1998).

the use of (4.6) in conjunction with (5.1), a deconvolution yields $T w$. By the use of (2.9) in conjunction with (5.2) with various assumptions and some convolutions and deconvolutions and a least-squares matching of shear stress profiles given by (2.9) and (5.2) a numerical result for $\beta_{a e}=\beta_{a e}[\Pi]$ was found.

However in the light of further work of Perry \& Marusic (1995) and Marusic \& Perry (1995) mainly concerned with problems with normal stress distributions, it was found necessary to use two structures, namely wall eddies, type A, and wake eddies, type B, as shown in figure 4. The vortex cores of type B do not extend to the wall but undulate in the spanwise direction. Although the wall attached eddies are capable of giving the correct shear stress distribution and mean velocity defect distribution, with the use of (5.1) and (5.2) they cannot reproduce the bumps at $\eta=0.5$ in the streamwise and spanwise components of the Reynolds stress distribution and at the same time give the correct distributions close to the wall. There are also difficulties with the component of Reynolds stress normal to the wall. See figures 5, 6 and 7 of Marusic \& Perry (1995). Wall eddies produce the pure wall flow as designated in equation (4.6) and the wake eddies produce the pure wake component as designated in (4.6). These two distinct eddy shapes were decided on after a long series of tests with all manner of eddy shapes. Type B eddies are responsible for the mean wake component of the velocity defect law and for the wake contribution to the Reynolds shear stress. The wall eddy contribution to the Reynolds shear stress is that given by what Coles (1957) calls pure wall flow, which is equivalent to (according to the authors' interpretation) $\beta=\beta_{a e}=-1 / 2 ; \Pi=0$ and $S \rightarrow \infty$ and this is substituted into (2.9) to obtain the wall eddy contribution of shear stress. Figure 4 shows this schematically. It should be noted here that pure wall flow occurs in equilibrium sink flow where $\mathrm{d} S / \mathrm{d} R_{x}=0$ and $\zeta=0$. It can be shown from (4.8) that $\beta=\beta_{a e}=-1 / 2$ as $S \rightarrow \infty$; see $\S 4.2$ of Jones et al. (2001) for details. Coles hypothesized that $\Pi=0$ also for this case. Although there is no proof for this, experiment certainly indicates that 
$\Pi$ approaches small values in sink flow (see Jones et al. 2001). The wake component of shear stress is obtained by subtracting the pure wall component as shown in figure $4(c)$.

Fortunately we need not elaborate on or make any commitments concerning the eddy shapes here. Hence

$$
\begin{aligned}
\frac{\mathrm{d} U_{D}^{*}}{\mathrm{~d} \lambda_{E}}=\frac{\mathrm{d} U_{D A}^{*}}{\mathrm{~d} \lambda_{E}}+\frac{\mathrm{d} U_{D B}^{*}}{\mathrm{~d} \lambda_{E}}= & \int_{-\infty}^{\infty} h_{A}[\lambda] \mathrm{e}^{-\lambda} T_{A}\left[\lambda-\lambda_{E}\right] w_{A}\left[\lambda-\lambda_{E}\right] \mathrm{d} \lambda \\
& +\int_{-\infty}^{\infty} h_{B}[\lambda] \mathrm{e}^{-\lambda} T_{B}\left[\lambda-\lambda_{E}\right] w_{B}\left[\lambda-\lambda_{E}\right] \mathrm{d} \lambda
\end{aligned}
$$

and

$$
\begin{aligned}
-\frac{\overline{u_{1} u_{3}}}{U_{\tau}^{2}}=-\left(\frac{\overline{u_{1} u_{3}}}{U_{\tau}^{2}}\right)_{A}-\left(\frac{\overline{u_{1} u_{3}}}{U_{\tau}^{2}}\right)_{B}= & \int_{-\infty}^{\infty}-J_{13 A}[\lambda] T_{A}^{2}\left[\lambda-\lambda_{E}\right] w_{A}\left[\lambda-\lambda_{E}\right] \mathrm{d} \lambda \\
& +\int_{-\infty}^{\infty}-J_{13 B}[\lambda] T_{B}^{2}\left[\lambda-\lambda_{E}\right] w_{B}\left[\lambda-\lambda_{E}\right] \mathrm{d} \lambda
\end{aligned}
$$

where $A$ and $B$ denote quantities associated with type A and type B eddies. The essential difference between this and the Perry et al. (1994) approach is the decomposition into two convolution integrals with two sets of weighting functions. We now subtract the contribution of type A eddies assuming that these are universal, i.e. independent of $\Pi$ and give pure wall flow, and the wall component of the Reynolds stress which is also universal, i.e. independent of $\Pi$.

Thus what follows is a consideration of type B eddies alone with $S \rightarrow \infty$ and $\zeta_{a}=0$. Hence

$$
\frac{\mathrm{d} U_{D B}^{*}}{\mathrm{~d} \lambda_{E}}=\int_{-\infty}^{\infty} h_{B}[\lambda] \mathrm{e}^{-\lambda} T_{B}\left[\lambda-\lambda_{E}\right] w_{B}\left[\lambda-\lambda_{E}\right] \mathrm{d} \lambda .
$$

Let us put

$$
T_{B}\left[\lambda-\lambda_{E}\right]=\widehat{T_{B}} \Psi[\xi], \quad w_{B}\left[\lambda-\lambda_{E}\right]=\widehat{w_{B}} \Phi[\xi],
$$

where $\widehat{T_{B}}$ and $\widehat{w_{B}}$ are weighting factor coefficients, $\xi=\lambda-\lambda_{E}=\ln \left(\delta / \delta_{c}\right)$ and $\Psi$ and $\Phi$ are assumed to be functions of $\xi$ alone. Experience with data and computations shows this form to be approximately true and a form of structural similarity is implied by this. Integrate (5.5) to give

$$
U_{D_{B}}^{*}[0]=\frac{2 \Pi}{\kappa}=\widehat{T_{B}} \widehat{w_{B}} \int_{0}^{1} \frac{1}{\eta} \int_{-\infty}^{\infty} h_{B}[\lambda] \mathrm{e}^{-\lambda} \Psi[\xi] \Phi[\xi] \mathrm{d} \lambda \mathrm{d} \eta ;
$$

the integral is a universal number $\mathscr{N}$ (say) and so

$$
\widehat{T_{B}} \widehat{w_{B}}=\frac{2 \Pi}{\kappa \mathscr{N}} .
$$

Now $h_{B}[\lambda] \mathrm{e}^{-\lambda}$ will have the general property of being zero at $\lambda= \pm \infty$ and $\Psi[\xi] \Phi[\xi]$ must be such that (5.7) converges. In the case of the wall component the corresponding equation to (5.7) does not converge since the defect law is logarithmic for $\eta \rightarrow 0$. Now the Reynolds shear stress wake component is given by

$$
-\left(\frac{\overline{u_{1} u_{3}}}{U_{\tau}^{2}}\right)_{B}=\int_{-\infty}^{\infty}-J_{13 B}[\lambda] T_{B}^{2}\left[\lambda-\lambda_{E}\right] w_{B}\left[\lambda-\lambda_{E}\right] \mathrm{d} \lambda .
$$


Integrate to give

$$
\int_{0}^{1}-\left(\frac{\overline{u_{1} u_{3}}}{U_{\tau}^{2}}\right)_{B} \mathrm{~d} \eta={\widehat{T_{B}}}^{2} \widehat{w_{B}} \int_{0}^{1} \int_{-\infty}^{\infty}-J_{13 B}[\lambda] \Psi^{2}[\xi] \Phi[\xi] \mathrm{d} \lambda \mathrm{d} \eta
$$

and so

$$
\int_{0}^{1}-\left(\frac{\overline{u_{1} u_{3}}}{U_{\tau}^{2}}\right)_{B} \mathrm{~d} \eta=M{\widehat{T_{B}}}^{2} \widehat{w_{B}}
$$

where $M$ is a universal number as given by the double integral on the right-hand side of equation (5.10).

It can be shown from (2.9) that for $S \rightarrow \infty$ after subtracting the pure wall component,

$$
\int_{0}^{1}\left(\frac{\tau}{\tau_{0}}\right)_{B} \mathrm{~d} \eta \approx \gamma\left(\beta_{a e}+1 / 2\right) .
$$

It has only a very weak dependence on $\Pi$ and $\gamma$ is a universal constant.

Hence from (5.11) and (5.12) we have

$$
\gamma\left(\beta_{a e}+1 / 2\right)=M{\widehat{T_{B}}}^{2} \widehat{w_{B}} .
$$

We now postulate (purely because it seems plausible and appears to agree with experiment) that

$$
\frac{{\widehat{T_{B}}}^{2}}{{\widehat{w_{B}}}^{2}}=P,
$$

where $P$ is a universal constant for all equilibrium layers, i.e. for all $\beta_{a e}$. If we assume power law relations, i.e.

$$
\widehat{T_{B}} \propto \Pi^{a}, \widehat{w_{B}} \propto \Pi^{b},
$$

then from (5.8) and (5.14) we obtain $a=1 / 3$ and $b=2 / 3$, i.e.

$$
{\widehat{T_{B}}}^{2} \widehat{w_{B}} \propto \Pi^{4 / 3},
$$

and from (5.11)

$$
\int_{0}^{1}-\left(\frac{\overline{u_{1} u_{3}}}{U_{\tau}^{2}}\right)_{B} \mathrm{~d} \eta=G \Pi^{4 / 3}
$$

where $G$ is a universal constant. Hence from (5.13)

$$
\beta_{a e}+1 / 2=Q \Pi^{4 / 3},
$$

where $Q$ is a universal constant. Figure 5 shows experimental data compared to (5.18) and it is seen that $Q=1.21$ represents a good fit. Therefore

$$
\beta_{a e}=-1 / 2+1.21 \Pi^{4 / 3}
$$

or

$$
\Pi=0.86\left(\beta_{a e}+1 / 2\right)^{3 / 4},
$$

which is very close to (3.5), the expression used by White (1974). It is interesting to note that the numerical values $-1 / 2$ and $4 / 3$ appearing in (5.19) come from theory. It is hoped that the simple expression given by (5.14) (which seems to work) can be combined with further knowledge to give a deeper physical insight. 


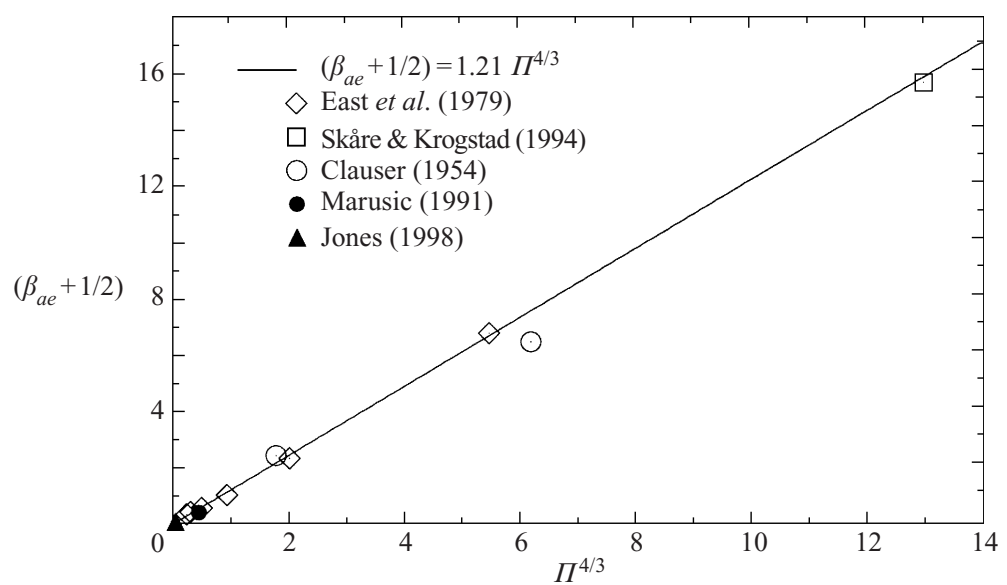

FIGURE 5. Experimental data that are in approximate equilibrium compared to (5.18).

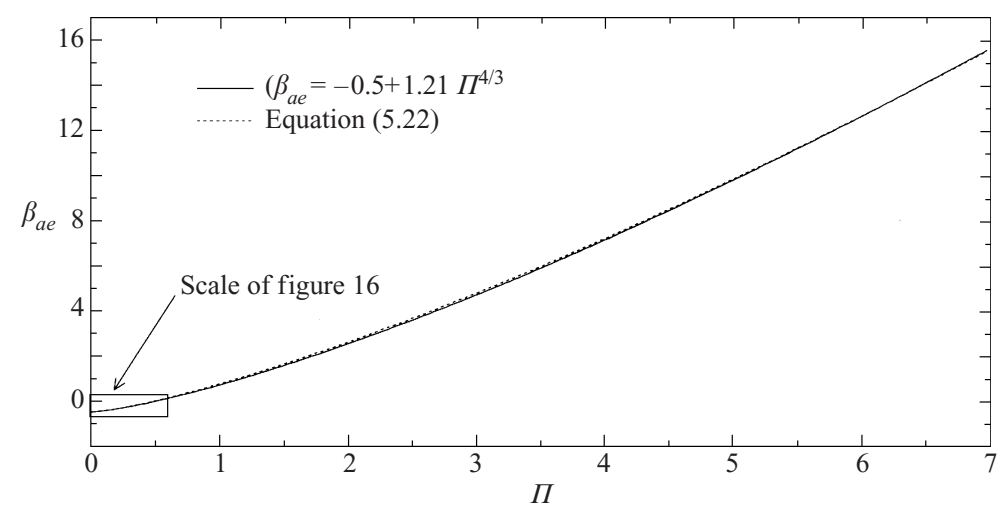

FIGURE 6. Equation (5.19) compared to (5.22) with $G=0.71$.

A more complete solution which avoids the approximation given by equation (5.12) can be found from (2.9) as follows:

$$
\int_{0}^{1}\left(\frac{\tau}{\tau_{0}}\right)_{B} \mathrm{~d} \eta=f_{1 i}[\Pi, \infty]-f_{1 i}[0, \infty]+g_{2 i}[\Pi, \infty] \beta_{a e}+g_{2 i}[0, \infty] \frac{1}{2} .
$$

Here the suffix $i$ denotes integration with respect to $\eta$ from 0 to 1 , and (5.21) is equal to $G \Pi^{4 / 3}$ from (5.17). In Appendix D it is shown from (2.9) that $g_{2 i}[\Pi, \infty]=f_{1 i}[\Pi, \infty]$ and that $g_{2 i}[0, \infty]=2 / 5$. Therefore

$$
\beta_{a e}=-1+\left(G \Pi^{4 / 3}+1 / 5\right) / g_{2 i}[\Pi, \infty] .
$$

If we make $G=0.71$ this is almost indistinguishable from (5.19) as seen in figure 6 . Therefore, we see that the attached eddy model can be used to fully describe the quasi-equilibrium flow problem where $\zeta_{a}=0$. Equations (5.19) and (5.22) are based on the presence of type B eddies. As $\Pi$ diminishes these eddies also diminish and so the theory behind (5.19) and (5.22) may break down because of the dominance of type A eddies.

The next step involves considering finite $\zeta_{a}$ effects. This is where dynamic effects are introduced into this extended attached eddy hypothesis. As a first attempt to do 
this, the appropriate term in (2.9) could be included so that (5.22) reads

$$
\beta_{a}=-1+\left(G \Pi^{4 / 3}-g_{1 i}[\Pi, \infty] \zeta_{a}+1 / 5\right) / g_{2 i}[\Pi, \infty] .
$$

Although (5.23) gives a family of curves for different $\zeta_{a}$ on the $\left(\Pi, \beta_{a}\right)$-plane, it is essentially equivalent to a universal distribution of eddy viscosity mentioned earlier. This is because the assumptions leading to equation (5.17) are consistent with all shear stress profiles requiring only $\Pi$ to be specified. This implies that $G$ is a constant. In reality $G$ could be a function of $\zeta_{a}$ and $\Pi$. This remains a topic of further study. One regime where things are likely to be different is in the case of very high $\Pi$ values.

\subsection{High $\Pi$ values}

The profile matching technique described in $\S 4$ is found to break down when $\Pi$ becomes very large (approx. $\Pi>5$ ) $\dagger$ with non-unique solutions for $\beta_{a}$ and $\zeta_{a}$ being possible. This situation is understood by examining equation (2.9) for $S \rightarrow \infty$ and $\Pi$ large. As shown in Appendix D the functions pre-multiplying $\zeta_{a}$ and $\beta_{a}$ are found to very closely resemble each other in shape and for $\Pi \rightarrow \infty$

$$
-g_{1}[\eta, \infty, \infty]=\frac{g_{2}[\eta, \infty, \infty]}{2 \kappa} .
$$

If this is the case, the shear stress profile shape is then determined by only one parameter, i.e.

$$
\beta_{a}-\frac{\zeta_{a}}{2 \kappa}
$$

In other words, any combination of $\beta_{a}$ and $\zeta_{a}$ may be valid provided the above parameter is fixed. Hence the profile matching method becomes ill-conditioned. More data are needed and perhaps more modelling so as to map out $\mathscr{F}[\Pi, S, \beta, \zeta]=0$. Then our computational procedure will work provided the log law of the wall and the law of the wake and the usual mean momentum and continuity equations are valid. Also, streamwise gradients of normal stresses must be negligible. When discussing separation, we need to ask how close to separation do we need to get? For Skåre \& Krogstad (1994) $\left(\tau / \tau_{0}\right) \max =17$ and $\Pi=6.85$ with $\zeta$ sufficiently small to neglect and these authors entitled their paper 'A turbulent boundary layer near 'separation'. These data are just outside the limit for accurate interpolation and extrapolation procedures. Most layers documented to be close to separation are best described as hovering in a state of incipient separation over long streamwise distances with the effects of $\zeta$ small. Quasi-equilibrium theory should hold and the streamwise gradients of normal stresses should be small. However, layers approaching separation with high $\zeta$, e.g. just upstream of a separation bubble, probably have large streamwise gradients of normal stresses and the problem is elliptic (see Perry \& Fairlie 1975), who approximated the approaching boundary layer as an inviscid region of constant vorticity with slip and used the Euler equations to successfully describe the vortical flow in and upstream of a separation bubble). Here boundary layer assumptions break down and the use of a prescribed external free-stream velocity is no longer an appropriate approach. Also, too close to any state of separation, the validity of the log law of the wall and the law of the wake becomes questionable (see Dengel \& Fernholz 1990). To

$\dagger$ The data of Skåre \& Krogstad (1994) in figure $5(\Pi=6.9)$ were obtained by assuming that the $\zeta=0$ contour in figure 2 was the same as for a trajectory of fixed shear stress distribution which passes a datum point of $\zeta=0$. This was approximately true for lower values of $\Pi$. 


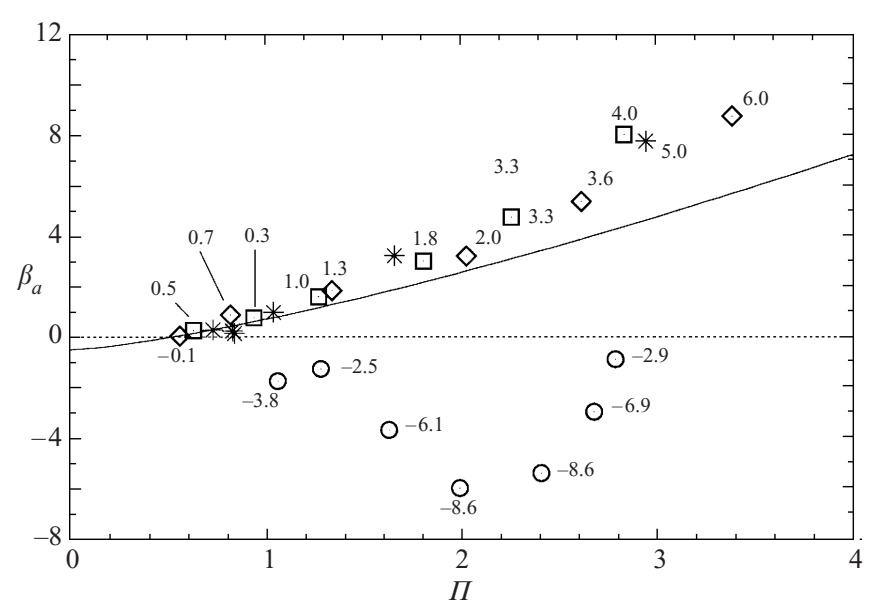

FIGURE 7. Comparison of equation (5.19) with non-equilibrium experimental data, i.e. with finite $\zeta$ values. Some typical values of $\zeta_{a}$ are shown for various data points. Data source: $\square$, Marusic \& Perry (1995) 30APG; O, Bradshaw \& Ferriss (1965); $\diamond$, Marusic \& Perry (1995) 10APG; *, Samuel \& Joubert (1974).

predict separation points together with separation bubbles, conventional boundary layer theory will not handle the problem no matter what closure model we use.

\section{Application to experiments}

\subsection{Closure equation}

In order to evaluate the evolution of the boundary layer a general expression is needed for (4.3), i.e.

$$
\psi\left[\Pi, \beta_{a}, \zeta_{a}\right]=0
$$

Although the attached eddy model can assist us for quasi-equilibrium flow, at the present time we still require a model for finite $\zeta_{a}$ values. The answer can be found empirically provided enough experimental information is available. A survey of existing experimental studies showed surprisingly how little reliable data are at present available. Moreover, in many cases the data in the literature failed the two-dimensional conservation of mean momentum test using (2.9). A collection of available results, believed to be the most reliable, covering a representative range of $\Pi, \beta_{a}, \zeta_{a}$ space is shown in figure 7. This plot represents the present state of experimental knowledge of closure of turbulent boundary layers and it can be seen to be very sparse. This is a rather disappointing state of affairs after over 40 years of data collection. All of these data had Reynolds shear stress data available where most profiles agreed reasonably well with equation (2.9). Using these data, a first tentative form for (4.3) has been estimated to be

$$
\Delta \beta_{a}= \begin{cases}\zeta_{a}^{2}\left(1.10 / \Pi^{2}\right) & \text { if } \quad \zeta_{a} \geqslant 0 \\ \zeta_{a}(0.62+0.25 \Pi) & \text { if } \quad \zeta_{a}<0\end{cases}
$$

Here

$$
\Delta \beta_{a}=\beta_{a}-\beta_{a e}
$$

where $\beta_{a e}$ is the value of $\beta_{a}$ for $\zeta_{a}=0$ and is given by (5.19) as obtained from the attached eddy model. Figure 8 shows plots of equation (6.1). It should be pointed out that quantities like $\beta_{a}$ and $\zeta_{a}$ were determined in part from $\beta$ and $\zeta$ which are 


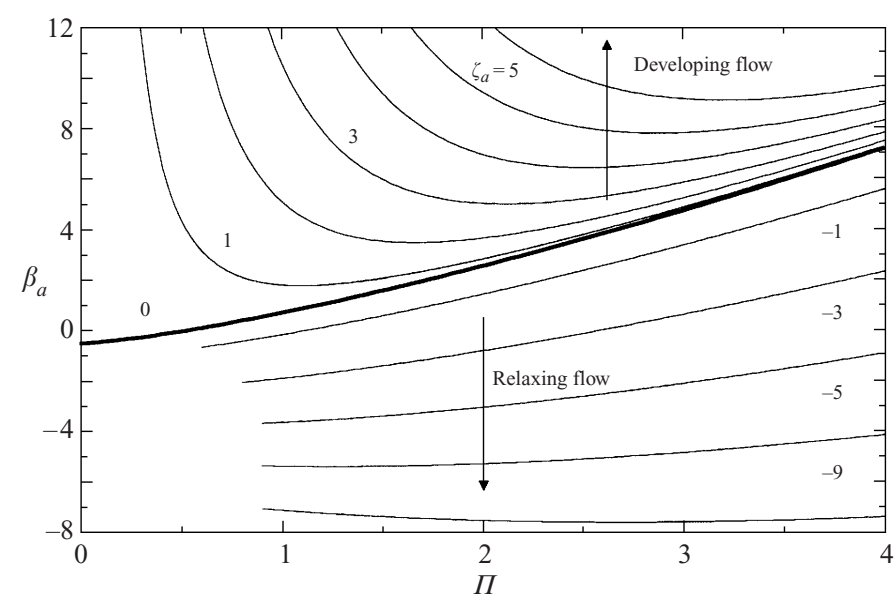

FIGURE 8. $\beta_{a}$ versus Coles wake factor for different $\zeta_{a}$ using (6.1) and (6.2).

found by differentiating curve fits to discrete data points. This is inherently inaccurate, particularly for $\zeta$ which was defined by very few points on a $\Pi$ versus $x$ plot. The quantity $\beta$ was more accurate since plots of $U_{1}$ versus $x$ had points including regions upstream and downstream of the last and first measuring stations. Inaccuracies in both $\beta_{a}$ and $\zeta_{a}$ are caused mainly by inaccuracies in $\zeta$. Thus (6.1) was found by perturbing the constants in the original surface fit to figure 7 until reasonable solutions to the ODEs were obtained, with the computed $\zeta$ in some cases differing from experiment but within a reasonable experimental error band. Reasonable results were obtained as can be seen from figure 9 , discussed later.

Given the evident sparseness of the experimental data, equation (6.1) can only be regarded as tentative. One interesting feature worth noting is the rapid change of the $\zeta_{a}$ surface slope $\mathrm{d} \zeta_{a} / \mathrm{d} \beta_{a}$ on the $\left(\beta_{a}, \Pi\right)$-plane at $\Delta \beta_{a}=0$. This might reflect the possibility that the processes in developing flows $\left(\zeta_{a}>0\right)$ and relaxing flows $\left(\zeta_{a}<0\right)$ are quite different physically. The use of the terms 'developing' and 'relaxing' defined by the sign of $\zeta$ was introduced by White (1974).

An early formulation by Perry, Marusic \& Jones (1998) is continuous at low $\Pi$ but a discontinuity in the slope of the $\zeta_{a}$ surface develops, i.e. a crease develops with increasing $\Pi$ along the $\zeta_{a}=0$ curve. The present formulation (6.1) has a crease for all $\Pi$ at $\zeta_{a}=0$. Perhaps one could formulate a continuous function for the slope at $\zeta_{a}=0$ by weighting the two expressions given in (6.1) so that one expression merges or morphs into the other as the $\zeta_{a}=0$ curve is crossed but we do not have sufficient data to do this with conviction. At $\Pi=2.5$ it is quite obvious that 'in the large' the slope of the $\zeta_{a}$ surface for fixed $\Pi$ is quite different for $\zeta_{a}>0$ and $\zeta_{a}<0$.

However there is another possible interpretation of all of this. Perhaps there will be cases which at finite $S$ have a positive $\zeta$ but when extrapolated to $S \rightarrow \infty$ with shear stress profile matching we obtain a $\zeta_{a}$ which is negative and vice versa. The sink flow cases in $\S 6.3 .1$ support this view. Hence perhaps we should define developing flow as flow with positive $\zeta$ as opposed to positive $\zeta_{a}$ and all such flows would give a $\left(\Pi, \beta_{a}\right)$-plane with contours of $\zeta_{a}$ both positive and negative and we conjecture $\zeta_{a}$ will be a regular function of $\beta_{a}$ at $\zeta_{a}=0$. We would then map out isosurfaces of $\zeta$ in the $S, \beta, \Pi$ space and denote it as developing flow. Any negative isosurface of $\zeta$ will have no physical meaning but will have mathematical meaning in the curve- and surface-fitting formulation. 
Similarly there would be a corresponding $\left(\Pi, \beta_{a}\right)$-plane with contours of $\zeta_{a}$ for relaxing flow where $\zeta$ is negative and again $\zeta_{a}$ will be positive and negative. There will be a corresponding $S, \beta, \Pi$ space with isosurfaces of $\zeta$ and the positive $\zeta$ will have only mathematical meaning. During the course of a computation there would be a switch from one space to the other if a developing flow becomes a relaxing flow. All of this is pure conjecture.

Here, we monitor $\zeta_{a}$ to decide which expression of (6.1) to use. This is equivalent to assuming that the $\zeta=0$ contour on the $(S, \beta)$-plane for fixed $\Pi$ is also a curve for fixed shear stress distribution. This is not quite true but in the philosophy of quasi-equilibrium theory it is approximately true. If this were precisely true then any data at finite $S$ with a positive $\zeta$ will always give a positive $\zeta_{a}$ with profile matching, and similarly a negative $\zeta$ will always yield a negative $\zeta_{a}$ with profile matching. There is another difficulty which needs to be explored in the future. With some trial formulations for (6.1) singularities have been seen to occur in the $S, \beta, \Pi$ space which we believe are physically unrealistic and should be removed by appropriate adjustment of (6.1). This might be a useful constraint on our choice of (6.1). There is no point in further serious development of (6.1) without more experimental data.

\subsection{Non-autonomous cases}

Equations (4.18) and (4.19) are applicable to the non-equilibrium turbulent boundary layer evolution. The independent variable $R_{x}$ appears explicitly on the right-hand side of the equations making them non-autonomous. This is what Clauser (1956) referred to as a black box with a clock inside. Autonomous cases will be studied in the next section.

Using (6.1) the evolution of several non-equilibrium flows were calculated given initial profiles and the results are shown in figure 9. The flows considered cover a broad distribution of adverse-pressure-gradient conditions. The flow of Bradshaw \& Ferriss (1965) is a relaxing flow, the two Marusic \& Perry (1995) cases are developing flows and the Samuel \& Joubert (1974) case has a short relaxing region followed by a developing region. Good agreement can be seen with experiment. This should be so since the data tested were used to formulate (6.1) in the first place. These calculations at least show that the scheme proposed is viable and the mathematics is working correctly.

Figures 10 and 11 show the computed shear stress profiles derived from the evolution results given in figure 9. These are compared with the measured Reynolds shear stress distributions and the results are very encouraging. In principle, Reynolds normal stresses and associated spectra and cross-power spectra could be computed for the fully turbulent part of the flow, i.e. $z_{+}>100$, using the convolution approach of Perry \& Marusic (1995) and Marusic \& Perry (1995). In fact this has already been done by Marusic \& Perry (1995) for their data presented here (see their figure 6). Of course further refinements would need to be made, such as the appropriate choice of representative eddy shapes and approximations to account for the Kolmogorov eddies (see Perry \& Marusic 1995; Marusic \& Perry 1995). In figure 11(b), the good agreement with experimental Reynolds shear stresses of the Bradshaw \& Ferriss (1965) stresses is considered fortuitous. Shear stress profiles generated by (2.9) when using experimental values of $S, \Pi, \beta$ and $\zeta$ are shown in figure $3(b)$. Agreement with results of figure 11 are not quite as good as the other cases. Thus there appears to be an anomality between measured stresses and overall momentum balances. Coles \& Hirst (1969) mention that there appeared to be some three-dimensional contamination. Nevertheless, this case is included since it is the best we have for relaxing flow. 


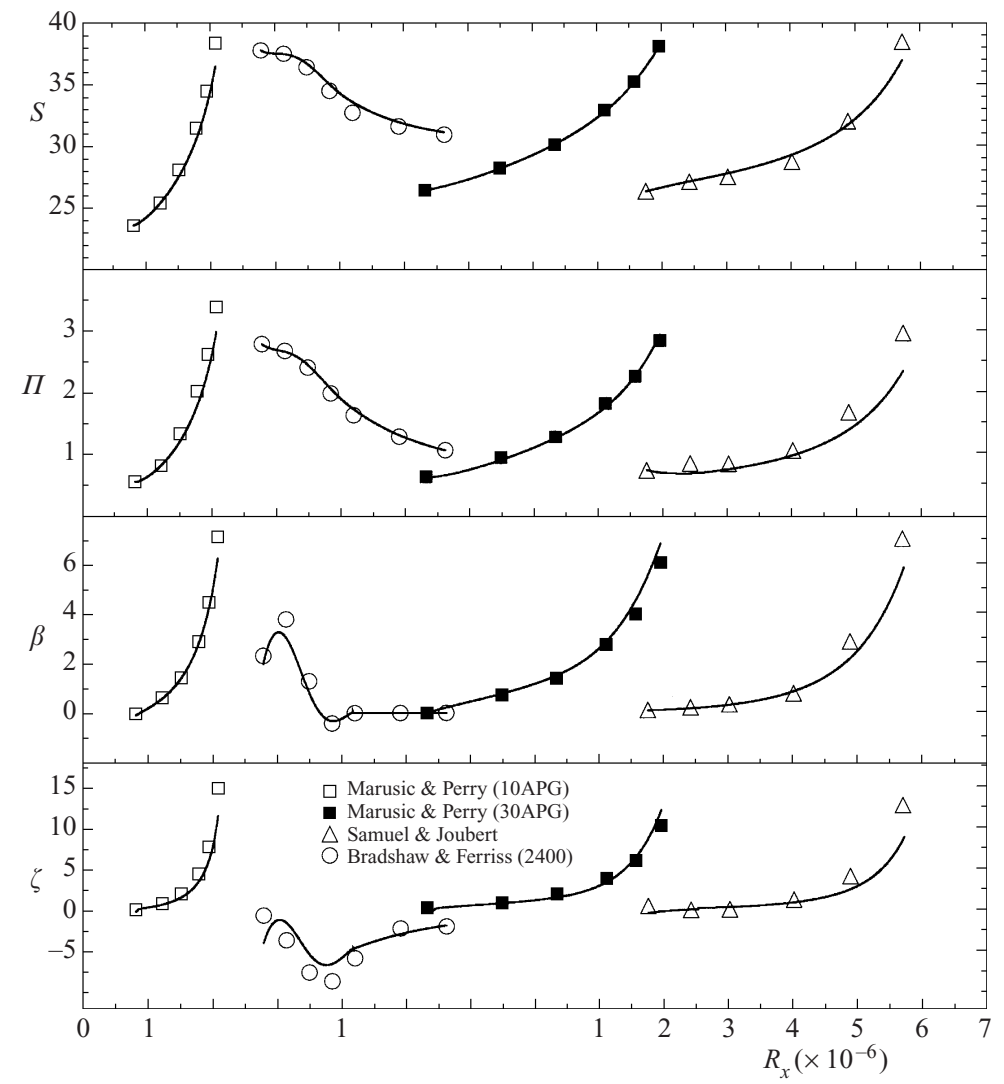

FIGURE 9. Non-equilibrium adverse pressure gradient data of Bradshaw \& Ferriss (1965), relaxing flow, Marusic \& Perry (1995), 30APG and 10APG flow, and Samuel \& Joubert (1974), positively increasing APG flow. Solid lines are the corresponding computed solutions of (4.8) and (4.9) using (4.10), (4.5) and (6.1), symbols are experimental values. The first $R_{x}$ scale belongs to $\square$ and $\bigcirc$, the second to $\mathbf{\square}$, the third to $\triangle$.

It should be noted that in solving the evolution equations, the position of $R_{x}=0$ is arbitrary. In all cases $R_{x}=0$ and $U_{1}=U_{0}$ was taken to occur at the trip wire. When integrating the ODEs, the first profile was always at a finite value of $R_{x}\left(R_{x}=R_{x_{i}}\right.$ say) and care was taken to ensure that $R_{\theta}$ was sufficiently large for the theory to be accurate at this initial Reynolds number $R_{x_{i}}$, see Appendix C Jones et al. (2001).

\subsection{Autonomous cases}

Clauser (1956) referred to this case as a black box without a clock inside. For flow cases such as zero pressure gradient, source flow or sink flow, $\left(1 / \chi^{2}\right)\left(\mathrm{d} \chi / \mathrm{d} R_{x}\right)$ in $(4.10)$ becomes a constant $K$, where for source flows

$$
K=\frac{-2 \pi v}{Q}=-\frac{1}{R_{L}},
$$

whereas for sink flows

$$
K=\frac{2 \pi v}{Q}=\frac{1}{R_{L}},
$$

and for zero-pressure-gradient flows $K=0$. Here $Q$ is the strength of the source 

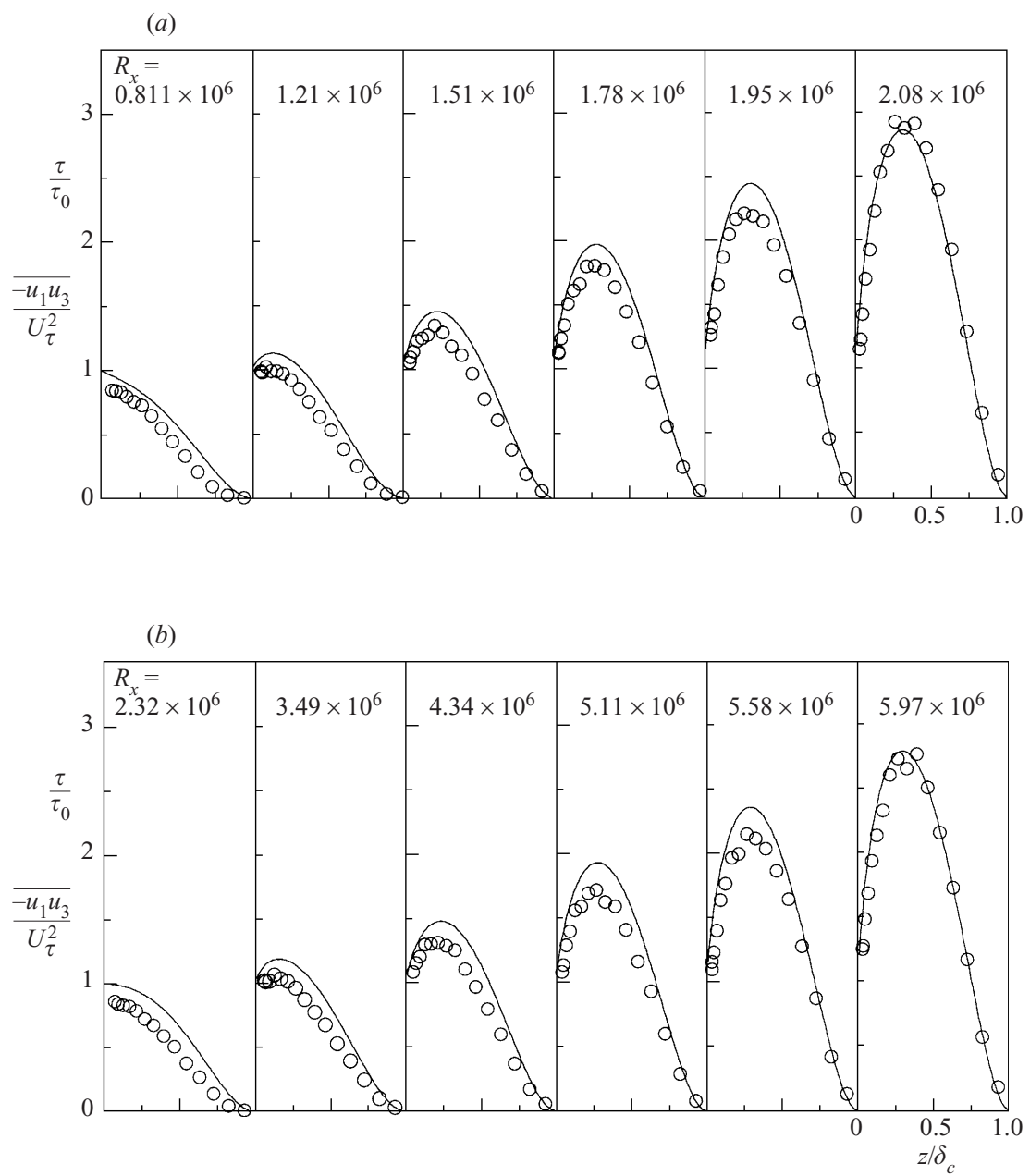

Figure 10. Reynolds shear stress data of Marusic \& Perry (1995) for (a) 10APG and (b) 30APG, compared to predicted evolution of the total shear stress from (2.9) using computed solutions of (4.8) and (4.9) with (4.10), (4.5) and (6.1). Profiles correspond with the data points in figure 9.

or sink (see figures 12 and 15 below). For these cases (4.18) and (4.19) become autonomous by an appropriate change in the independent variable $R_{x}$ to $T_{x}$ to give

$$
\begin{aligned}
& \frac{\mathrm{d} S}{\mathrm{~d} T_{x}}=\psi_{1}[\Pi, S, K], \\
& \frac{\mathrm{d} \Pi}{\mathrm{d} T_{x}}=\psi_{2}[\Pi, S, K] .
\end{aligned}
$$

where $T_{x}=-\left(\ln \left(1-R_{x} K\right)\right) / K$. Also, in the limit as $K \rightarrow 0$ (i.e. zero-pressuregradient flow) $T_{x}=R_{x}$. This means that solution trajectories can be displayed on the $(S, \Pi)$-phase plane for various initial conditions for fixed $K$.

Figure 12 shows a schematic representation of source flow and figure 13 shows calculations for a series of different initial conditions. As mentioned earlier, this is an autonomous system and so the solution can be displayed on a $(S, \Pi)$-phase-plane diagram. There is no experimental data for this case so it represents a genuine 

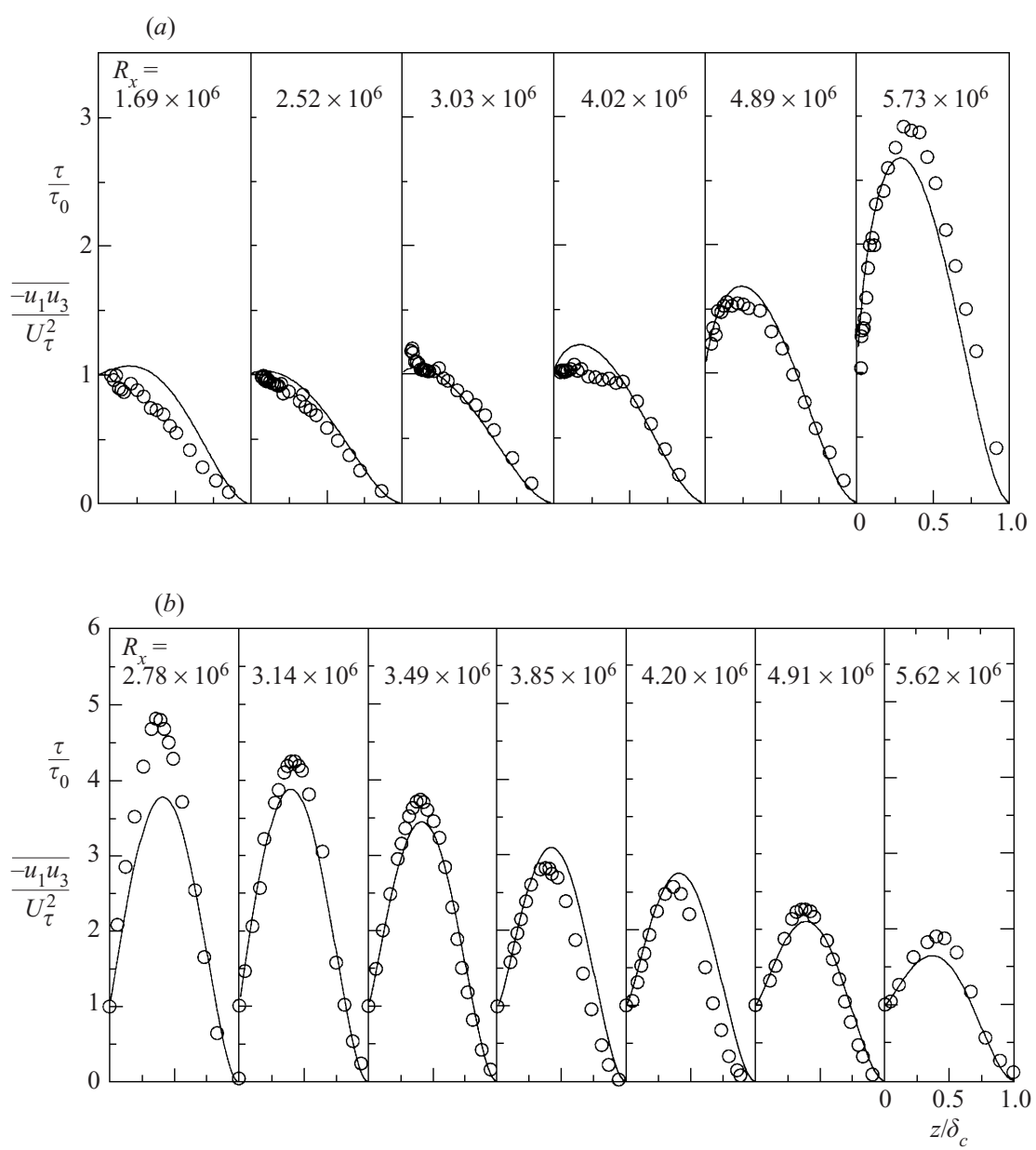

Figure 11. Reynolds shear stress data of (a) Samuel \& Joubert (1974) and (b) Bradshaw \& Ferriss (1965), compared to predicted evolution of the total shear stress from (2.9) using computed solutions of (4.8) and (4.9) with (4.10), (4.5) and (6.1). Profiles correspond with the data points in figure 9.

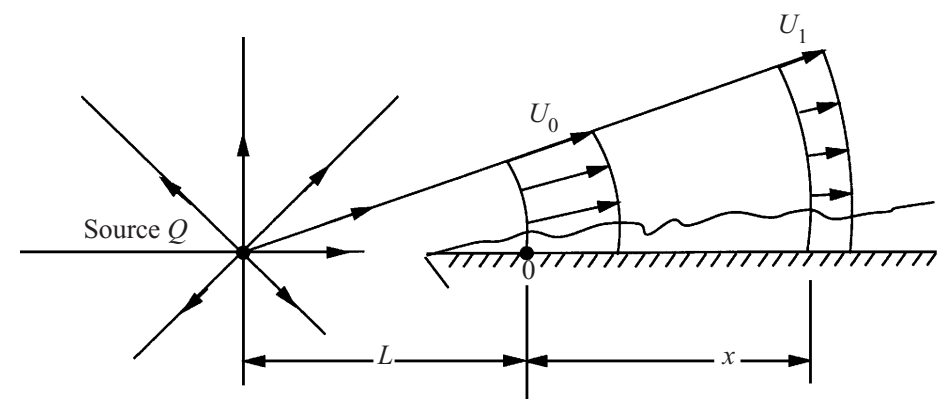

FIGURE 12. Schematic of source flow boundary layer; note that the origin for $x$ is arbitrary.

prediction. The range of validity of (6.1) is very limited in $\zeta_{a}$ and this may have been exceeded in computing figure 13 . However whether the intriguing pattern shown in figure 13 is realistic cannot be known due to a complete lack of experimental data. The heavy broken line in figure 13 is from the Perry et al. (1994) quasi-equilibrium 


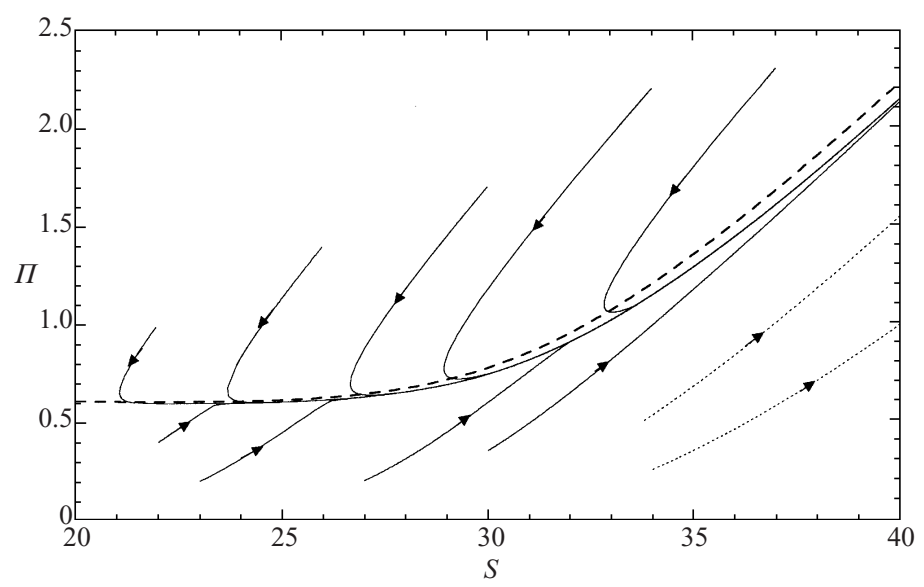

FIGURE 13. Source flow phase-plane trajectory calculations for $K=v /\left(L U_{0}\right)=-10^{-8}$. Heavy broken line is the quasi-equilibrium solution of Perry et al. (1994).

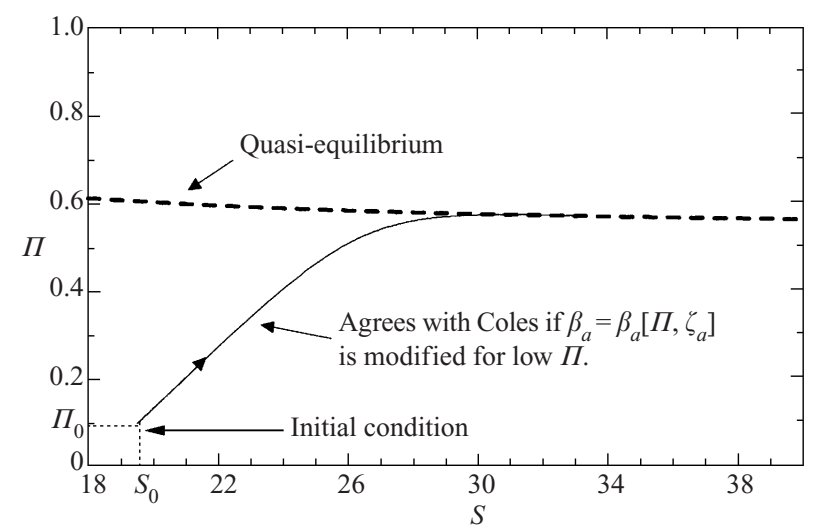

FIGURE 14. Zero pressure gradient phase plane trajectory, after Perry et al. (1998), where the Lewkowicz (1982) formulation was used.

calculation using (5.19) for $\beta_{a e}$. It can be seen that for that calculation we are not free to choose the initial conditions for $\Pi$ and $S$ independently.

In figure 14 is shown the case of a zero-pressure-gradient flow as calculated by Perry et al. (1998), using preliminary versions of (5.20) and (6.1). It can be seen that this layer rapidly asymptotes into the quasi-equilibrium layer of Perry et al. (1994) and becomes a true equilibrium layer only at infinite Reynolds numbers. The initial condition are chosen to correspond to Coles (1962) 'standard trip' development at a value of $R_{\theta}$ sufficiently large for the theory to be accurate. Other initial conditions should be systematically studied experimentally. What this formulation implies is that a zero-pressure-gradient layer is not an equilibrium layer but it is an autonomous layer which rapidly asymptotes into a quasi-equilibrium layer and reaches a state of precise equilibrium (self-similarity) only at infinite Reynolds numbers. Under these conditions it is simple to show that the evolution equations yield the analytical form of the Kármán law of skin friction (see Perry 1992). 


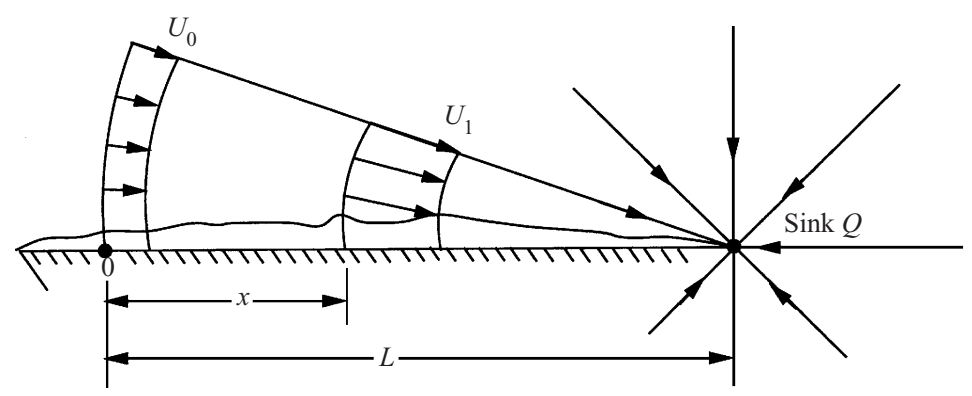

FIGURE 15 . Schematic of sink flow boundary layer; note that the origin for $x$ is arbitrary.

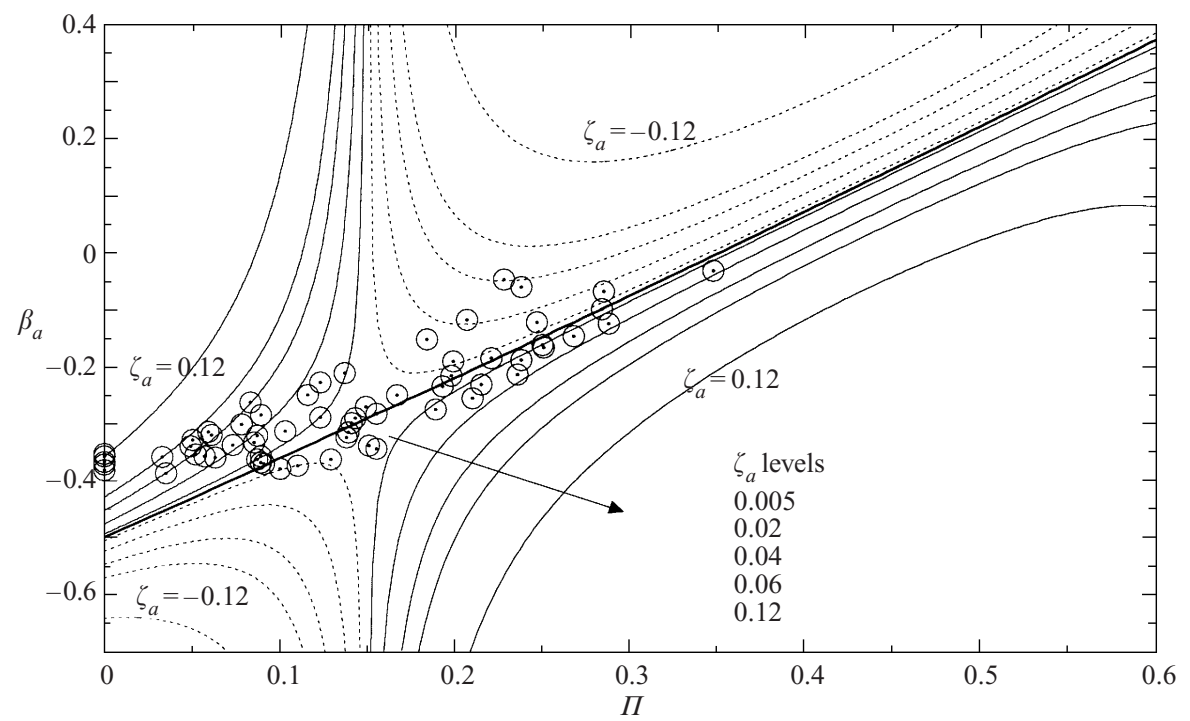

FiguRE 16. Data of Jones (1998). Each point has a different value of $\zeta_{a}$. Solid line corresponds to equation (6.5) for $\zeta_{a}=0$. The curve fits are localized and the extent of the plot is shown in figure 6 for comparison.

\subsubsection{Approaching sink flow}

A recent thorough study of sink flows was carried out by Jones (1998) and Jones et al. (2001) and these are also considered here. Such flows are a good example of favourable-pressure-gradient flows. A sink-flow turbulent boundary layer is one whose pressure gradient follows that of a two-dimensional potential sink. The flow is shown schematically in figure 15 . Townsend (1956) and Rotta (1962) identified sink flow as the only smooth wall boundary layer that may evolve to a state of precise equilibrium at finite $S$ for flows which are two-dimensional in the mean. A precise equilibrium layer is one where all mean and turbulence measurements are invariant with streamwise distance, when they are scaled with the correct velocity and length scales. In this experimental study, three acceleration parameters $(K)$ were investigated with a total of 62 mean flow stations. Figure 16 shows the corresponding $\beta_{a}$ versus $\Pi$ data. Expression (6.1) is not expected to apply in this range of $\Pi$.

The heavy solid line in figure 16 corresponds to a curve fit for the $\zeta_{a}=0$ data points with the functional form

$$
\beta_{a e}=-0.5+1.38 \Pi+0.13 \Pi^{2} .
$$



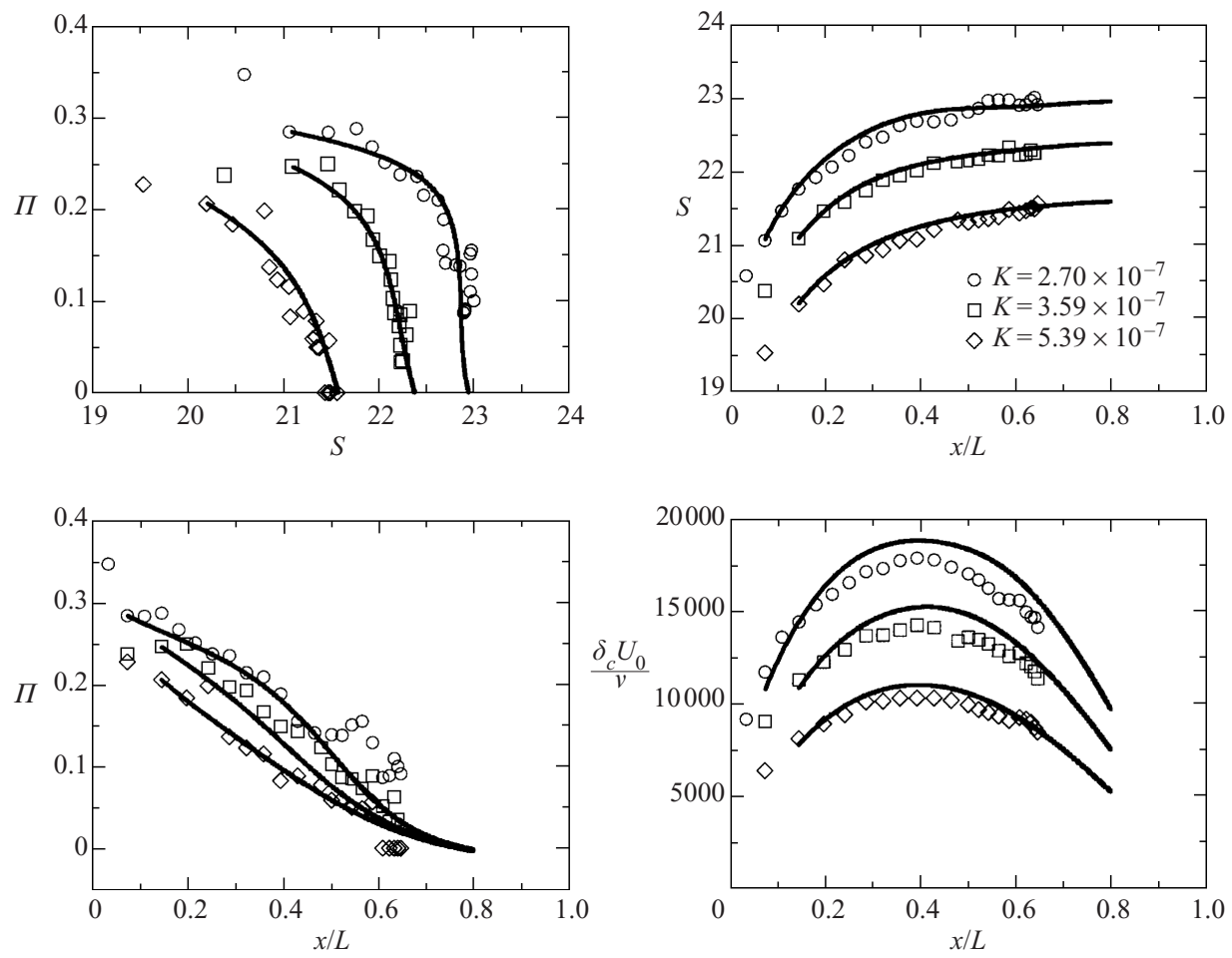

FiguRE 17. Evolution of mean flow parameters for flow of Jones (1998), see also Jones et al. (2001). Symbols represent data; solid lines correspond to calculation. Note in these figures that $x$ is measured from the trip wire.

Equations (6.5) and (5.19) are very close for $\Pi$ greater than about 2. Data show that (6.5) is more valid for low $\Pi$ (i.e. $\Pi<0.3$ ) than (5.19) and (5.9). To find how these curves merge and the associated contours of $\zeta_{a}$ will require more data. The lines of constant $\zeta_{a}$ shown in figure 16 come from a localized surface fit:

$$
\zeta_{a}=\left(0.85-6.9 \Pi+8 \Pi^{2}\right) \Delta \beta_{a} .
$$

Computations were carried out without monitoring the sign of $\zeta_{a}$ since (6.6) is regular (see discussion in $§ 6.1$ ).

Using (6.6), (4.10) and the least-squares-error shear stress profile matching of (4.5), formulation (4.1) can be described and thus equations (4.8) and (4.9) can now be put into the form of (6.3) and (6.4) and we can compute the evolution of the boundary layer given any initial station where $\Pi$ and $S$ are known. Figure 17 shows a comparison between the experimental data and computation and good agreement is observed. This shows that the closure scheme is a viable one and indicates that the mathematics is working correctly. Figure 18 shows the progressive improvement in the description of the streamwise evolution of $\beta$. In figure 18(a) the result of a quasiequilibrium formulation using the Green, Weeks \& Brooman (1973) formula is shown, figure $18(b)$ is a quasi-equilibrium calculation using (6.5) and finally figure $18(c)$ is the non-equilibrium calculation using (6.5) and (6.6).

To help determine the range in which equation (6.6) might be extended, a series of initial conditions were tried for the evolution of sink flow for a given $K$ value. The results are shown in figure 19. As mentioned earlier, with sink flow the governing 


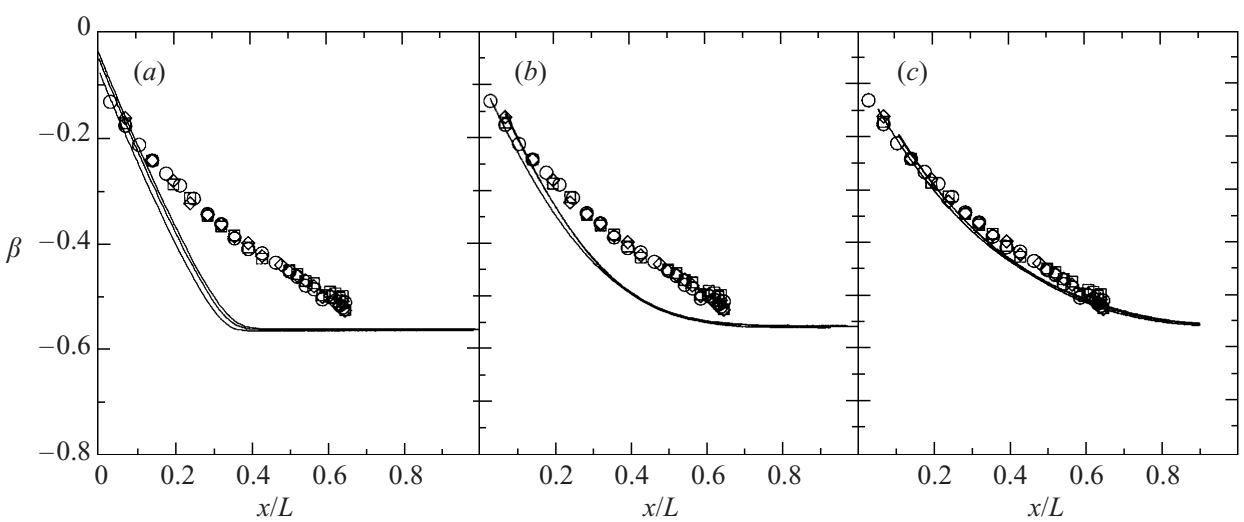

FIGURE 18. Evolution of $\beta$ for flow of Jones (1998). Three evolution calculations are shown, corresponding to: (a) quasi-equilibrium calculation using the Green et al. (1973) formula, (b) quasi-equilibrium calculation using (6.5) and $(c)$ the full non-equilibrium calculation using (6.5) and (6.6).

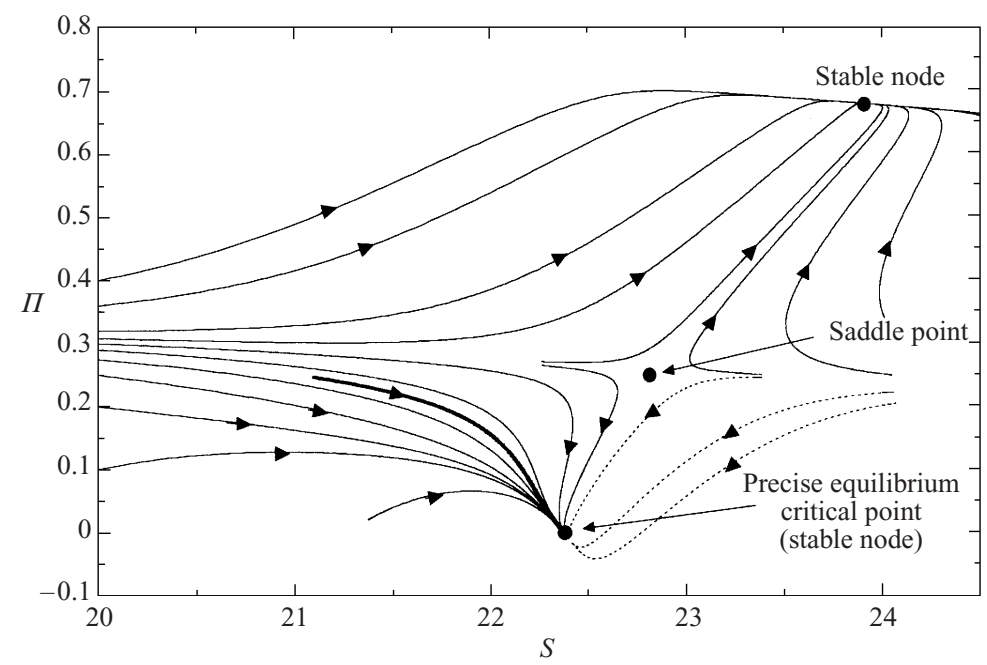

FIGURE 19. Phase-plane solution trajectories for sink flow $\left(K=3.59 \times 10^{-7}\right)$ starting from different initial conditions. Heavy line trajectory corresponds to flow case in figure 17.

equations are autonomous. This means that the $(S, \Pi)$-plane is a phase plane and solution trajectories can only cross at critical points. As can be seen in the figure, solution trajectories converge to an equilibrium solution as expected, i.e. a stable node critical point, but we observe that two such critical points and a saddle point exist between these two stable nodes. The $\Pi \approx 0$ solution is the expected one and the other would seem to result when using equation (6.6) beyond its range of applicability.

As in the quasi-equilibrium calculations of Jones et al. (2001), these more complete non-equilibrium equations predict precise equilibrium at finite $S$ with $\Pi$ at a small but positive value and $\beta$ slightly more negative than $-1 / 2$. Because $\Pi$ is very small (i.e. virtually pure wall flow) it seems that there exists not one universal pure wall flow with $S=\infty$ and $\beta=-1 / 2$ but a whole family of pure wall shear stress profiles characterized by $S$. This may be important when applying the attached eddy hypothesis of Perry \& Marusic (1995) at finite $S$. 


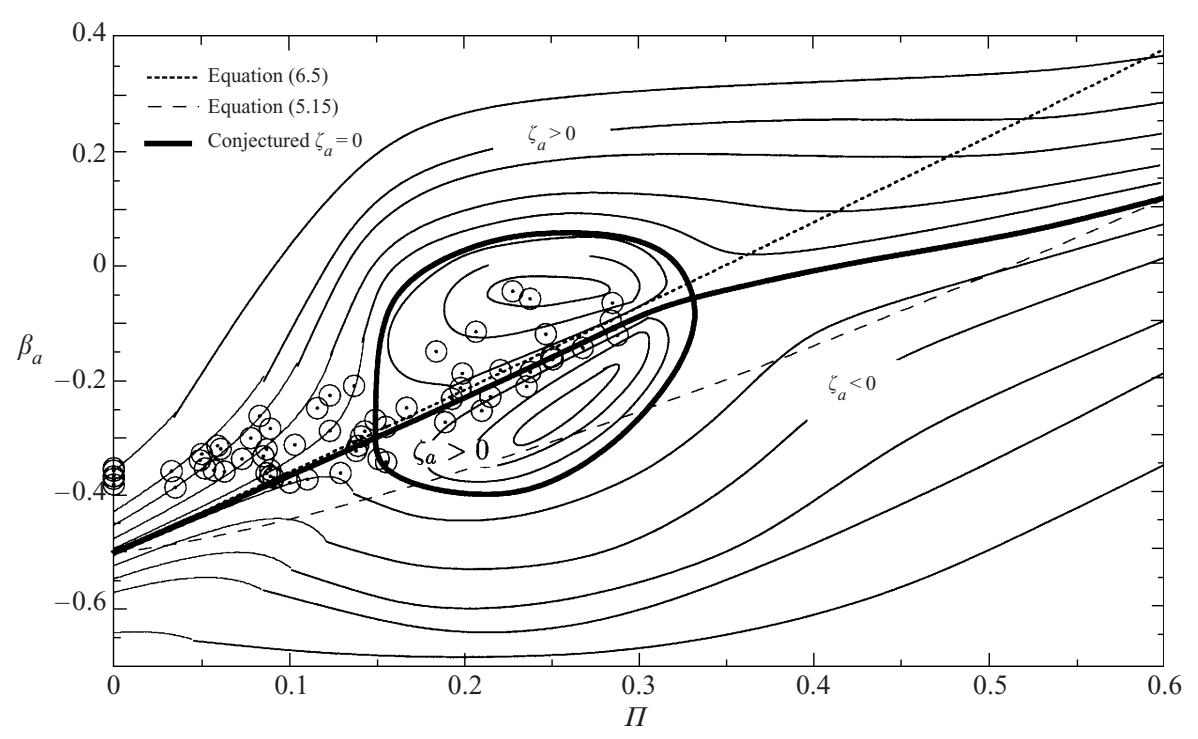

FIGURE 20. Conjectured $\beta_{a}$ for combining the contours of figure 8 with 16 .

\section{Conclusions and discussion}

This closure scheme is based on the log law of the wall, law of the wake, the mean continuity and momentum differential equations and the momentum integral equation with the usual boundary layer approximations and neglecting the streamwise gradients of the normal stresses. Analysis shows that the mean velocity profiles and total shear stress profiles are completely described by the four parameters $S, \Pi, \beta$ and $\zeta$ as first suggested by Perry et al. (1994). It is further assumed that there is a universal relation of the form $\mathscr{F}[\Pi, S, \beta, \zeta]=0$. This function has to be determined from experimental data. Once this is done closure is complete. However because of the sparseness of the data some aids are required for interpolation and extrapolation. One aid is based on the assumption that the shear stress profiles form a two-parameter family as opposed to the one-parameter family implied in many eddy viscosity models. By shear stress matching on this basis, results were interpolated and extrapolated. As a further aid, the attached eddy hypothesis was used for forming a semi-theoretical relation between $\beta_{a}$ and $\Pi$ for $\zeta_{a}=0$. This modelling should be extended to include finite values of $\zeta_{a}$.

Evolution equations based on an integral approach are consistent with the attached eddy hypothesis which utilizes convolution integrals. The above approach seems more physically reasonable than most differential field methods because in reality the transport properties at one point in the flow must be intimately related to motion remote from that point. This feature is an important aspect of the attached eddy hypothesis.

The proposed formulations for $\psi\left[\Pi, \beta_{a}, \zeta_{a}\right]$ are very tentative and the variation in its form between cases of moderate $\Pi$ and very low $\Pi$ gives some idea of the complexity that may be involved in one general formulation covering all flows. From an inspection of figures 8 and 16, positive and negative $\zeta_{a}$ contours at low $\Pi$ have to be accommodated with the corresponding contours of $\zeta_{a}$ at the higher $\Pi$. As Clauser pointed out in his insightful 1956 paper when discussing such surfaces in an appropriate multi-dimensional space, 'The surface need not be continuous nor single 
valued. It may be difficult to determine, but, in principle, sufficient experimentation can eventually plot out the entire surface'. The degree of complexity (or simplicity) will not be known until this data is mapped out completely. A conjectured possibility for combining the contours of figures 8 and 16 is given in figure 20 .

The aim of the present work is to describe a possible framework for computing streamwise evolution and assess its viability. The viability of the scheme has been demonstrated here and further experimental data would be needed so that a single formulation covering a broad range of parameters can be developed. The use of models such as those based on the attached eddy hypothesis show potential and should with further development be useful in helping to map out the $\Pi, \beta_{a}, \zeta_{a}$ space with appropriate interpolation and extrapolation schemes. Once the mean flow development can be calculated there is the potential to apply the attached eddy model of Perry \& Marusic (1995) to evaluate the relevant turbulence quantities, such as Reynolds normal stresses and spectra.

The authors wish to acknowledge the financial assistance of the Australian Research Council.

\section{Appendix A. Shear stress distribution}

From (2.1) to (2.7) we have

$$
\frac{\tau}{\tau_{0}}=1+A_{1} X_{1}+A_{2} X_{2}+A_{3} X_{3}+A_{4} X_{4}
$$

where

$$
X_{1}=\frac{\mathrm{d} \delta_{c}}{\mathrm{~d} x}, \quad X_{2}=\frac{\delta_{c}}{S} \frac{\mathrm{d} S}{\mathrm{~d} x}, \quad X_{3}=\delta_{c} \frac{\mathrm{d} \Pi}{\mathrm{d} x}, \quad X_{4}=\frac{\delta_{c}}{U_{1}} \frac{\mathrm{d} U_{1}}{\mathrm{~d} x},
$$

and

$$
\begin{aligned}
& A_{1}=\int_{0}^{\eta} f^{2} \mathrm{~d} \eta-f \int_{0}^{\eta} f \mathrm{~d} \eta+S\left(\eta f-\int_{0}^{\eta} f \mathrm{~d} \eta\right), \\
& A_{2}=-2 \int_{0}^{\eta} f^{2} \mathrm{~d} \eta+S \int_{0}^{\eta} f \mathrm{~d} \eta+f \int_{0}^{\eta} f \mathrm{~d} \eta, \\
& A_{3}=\frac{\mathrm{d}}{\mathrm{d} \Pi} \int_{0}^{\eta} f^{2} \mathrm{~d} \eta-(f+S) \frac{\mathrm{d}}{\mathrm{d} \Pi} \int_{0}^{\eta} f \mathrm{~d} \eta, \\
& A_{4}=2 \int_{0}^{\eta} f^{2} \mathrm{~d} \eta-f \int_{0}^{\eta} f \mathrm{~d} \eta+S\left(\eta f-3 \int_{0}^{\eta} f \mathrm{~d} \eta\right) .
\end{aligned}
$$

There exists relationships between the $X_{i}$ parameters which can be established by considering the outer boundary conditions. The first boundary condition can be established from the law of the wall and law of the wake with the outer boundary condition $U=U_{1}$; at $z=\delta_{c}$ this gives

$$
S=\frac{U_{1}}{U_{\tau}}=\frac{1}{\kappa} \ln \left[\frac{\delta_{c} U_{\tau}}{v}\right]+A+\frac{\Pi}{\kappa} W_{c}[1, \Pi] .
$$

Then differentiating the above with respect to $x$ it can be shown that

$$
X_{2}(\kappa S+1)=X_{4}+X_{1}+N X_{3}
$$

where

$$
N=W_{c}[1, \Pi]+\Pi \frac{\mathrm{d} W_{c}[1, \Pi]}{\mathrm{d} \Pi}=2 .
$$


A further outer boundary condition exists, namely $\tau / \tau_{0}=0$ at $\eta=1$, and from (A 1) this gives

$$
0=1+B_{1} X_{1}+B_{2} X_{2}+B_{3} X_{3}+B_{4} X_{4}
$$

where $B_{i}=A_{i}[1, \Pi, S]$. Equations (A 3) and (A 4) are used to eliminate $X_{1}$ and $X_{2}$ from the shear stress expression given in (A 1):

$$
\frac{\tau}{\tau_{0}}=f_{1}[\eta, \Pi, S]+f_{2}[\eta, \Pi, S] \delta_{c} \frac{\mathrm{d} \Pi}{\mathrm{d} x}+f_{3}[\eta, \Pi, S] \frac{\delta_{c}}{U_{1}} \frac{d U_{1}}{\mathrm{~d} x}
$$

where

$$
\left.\begin{array}{l}
f_{1}=1-\frac{A_{1}}{B_{1}+E_{1} B_{2}}-\frac{E_{1} A_{2}}{B_{1}+E_{1} B_{2}}, \\
f_{2}=\frac{E_{1} N A_{2} B_{1}+A_{3} B_{1}-E_{1} N A_{1} B_{2}+E_{1} A_{3} B_{2}-A_{1} B_{3}-E_{1} A_{2} B_{3}}{B_{1}+E_{1} B_{2}}, \\
f_{3}=\frac{E_{1} A_{2} B_{1}+A_{4} B_{1}-E_{1} A_{1} B_{2}-E_{1} A_{4} B_{2}-A_{1} B_{4}-E_{1} A_{2} B_{4}}{B_{1}+E_{1} B_{2}},
\end{array}\right\}
$$

and $E_{1}=1 /(1+\kappa S)$.

It is often more convenient to write (A 5) as

$$
\frac{\tau}{\tau_{0}}=f_{1}[\eta, \Pi, S]+g_{1}[\eta, \Pi, S] \zeta+g_{2}[\eta, \Pi, S] \beta,
$$

where $g_{1}=f_{2} / S, g_{2}=-f_{3} /\left(C_{1} S\right)$.

The structures of the functions in (A 7) are

$$
\left.\begin{array}{l}
f_{1}[\eta, \Pi, S]=\frac{a_{1} S^{2}+a_{2} S+a_{3}}{b_{1} S^{2}+b_{2} S+b_{3}}, \\
g_{1}[\eta, \Pi, S]=\frac{c_{1} S^{3}+c_{2} S^{2}+c_{3} S+c_{4}}{S\left(e_{1} S^{2}+e_{2} S+e_{3}\right)}, \\
g_{2}[\eta, \Pi, S]=\frac{q_{1} S^{3}+q_{2} S^{2}+q_{3} S+q_{4}}{S\left(r_{1} S^{2}+r_{2} S+r_{3}\right)},
\end{array}\right\}
$$

where $a_{1}, a_{2}$ etc. are polynomials in $\eta, \log \eta$ and $\Pi$.

\section{Appendix B. Evolution equations}

The momentum integral equation is given by

$$
\frac{\mathrm{d} \theta}{\mathrm{d} x}+\frac{(H+2) \theta}{U_{1}} \frac{\mathrm{d} U_{1}}{\mathrm{~d} x}=\frac{C_{f}^{\prime}}{2},
$$

where $C_{f}^{\prime} / 2=1 / S^{2}$. Using the defect function given in (2.6) in conjunction with the wake expression contained in (4.6) expressions for the momentum and displacement thickness are

$$
\begin{gathered}
\theta=\delta_{c}\left(\frac{C_{1}[\Pi]}{S}-\frac{C_{2}[\Pi]}{S^{2}}\right), \\
\delta^{*}=\frac{\delta_{c} C_{1}[\Pi]}{S},
\end{gathered}
$$


from which the terms appearing on the left-hand side of (B 1) can be expressed as

$$
\begin{aligned}
\frac{\mathrm{d} \theta}{\mathrm{d} x}= & \frac{\mathrm{d} \delta_{c}}{\mathrm{~d} x}\left(\frac{C_{1}[\Pi]}{S}-\frac{C_{2}[\Pi]}{S^{2}}\right)+\delta_{c}\left(\frac{\mathrm{d} C_{1}[\Pi]}{\mathrm{d} \Pi} \frac{\mathrm{d} \Pi}{\mathrm{d} x} \frac{1}{S}-\frac{\mathrm{d} C_{2}[\Pi]}{\mathrm{d} \Pi} \frac{\mathrm{d} \Pi}{\mathrm{d} x} \frac{1}{S^{2}}\right) \\
& +\delta_{c}\left(\frac{-C_{1}[\Pi]}{S^{2}} \frac{\mathrm{d} S}{\mathrm{~d} x}+\frac{2 C_{2}[\Pi]}{S^{3}} \frac{\mathrm{d} S}{\mathrm{~d} x}\right)
\end{aligned}
$$

and

$$
\frac{(H+2) \theta}{U_{1}} \frac{\mathrm{d} U_{1}}{\mathrm{~d} x}=\frac{-\beta}{C_{1}[\Pi] S}\left(\frac{3 C_{1}[\Pi]}{S}-\frac{2 C_{2}[\Pi]}{S^{2}}\right) .
$$

Using the outer boundary condition given in (A 2) gives

$$
\delta_{c}=\frac{v}{U_{\tau}} E[\Pi] \exp (\kappa S)
$$

and hence

$$
\frac{\mathrm{d} \delta_{c}}{\mathrm{~d} x}=\kappa \delta_{c} \frac{\mathrm{d} S}{\mathrm{~d} x}-\frac{\delta_{c}}{U_{\tau}} \frac{\mathrm{d} U_{\tau}}{\mathrm{d} x}-\delta_{c} N \frac{\mathrm{d} \Pi}{\mathrm{d} x} .
$$

Substituting (B 7) into (B 4) gives

$$
\begin{aligned}
\frac{\mathrm{d} \theta}{\mathrm{d} x}= & \delta_{c}\left(\kappa \frac{\mathrm{d} S}{\mathrm{~d} x}-\frac{1}{U_{\tau}} \frac{\mathrm{d} U_{\tau}}{\mathrm{d} x}-N \frac{\mathrm{d} \Pi}{\mathrm{d} x}\right)\left(\frac{C_{1}[\Pi]}{S}-\frac{C_{2}[\Pi]}{S^{2}}\right) \\
& +\delta_{c}\left(\frac{\mathrm{d} C_{1}[\Pi]}{\mathrm{d} \Pi} \frac{\mathrm{d} \Pi}{\mathrm{d} x} \frac{1}{S}-\frac{\mathrm{d} C_{2}[\Pi]}{\mathrm{d} \Pi} \frac{\mathrm{d} \Pi}{\mathrm{d} x} \frac{1}{S^{2}}\right)+\delta_{c}\left(\frac{-C_{1}[\Pi]}{S^{2}} \frac{\mathrm{d} S}{\mathrm{~d} x}+\frac{2 C_{2}[\Pi]}{S^{3}} \frac{\mathrm{d} S}{\mathrm{~d} x}\right) .
\end{aligned}
$$

Putting

and noting

$$
\zeta=S \delta_{c} \mathrm{~d} \Pi / \mathrm{d} x
$$

gives

$$
\frac{\delta_{c}}{U_{\tau}} \frac{\mathrm{d} U_{\tau}}{\mathrm{d} x}=-\frac{\beta}{S C_{1}[\Pi]}-\frac{\delta_{c}}{S} \frac{\mathrm{d} S}{\mathrm{~d} x}
$$

$$
\begin{aligned}
\frac{\mathrm{d} \theta}{\mathrm{d} x}= & \delta_{c} \frac{\mathrm{d} S}{\mathrm{~d} x}\left\{\kappa\left(\frac{C_{1}[\Pi]}{S}-\frac{C_{2}[\Pi]}{S^{2}}\right)+\frac{C_{2}[\Pi]}{S^{3}}\right\}+\frac{\beta}{S C_{1}[\Pi]}\left(\frac{C_{1}[\Pi]}{S}-\frac{C_{2}[\Pi]}{S^{2}}\right) \\
& +\frac{\zeta}{S}\left\{-N\left(\frac{C_{1}[\Pi]}{S}-\frac{C_{2}[\Pi]}{S^{2}}\right)+\frac{1}{S} \frac{\mathrm{d} C_{1}[\Pi]}{\mathrm{d} \Pi}-\frac{1}{S^{2}} \frac{\mathrm{d} C_{2}[\Pi]}{\mathrm{d} \Pi}\right\} .
\end{aligned}
$$

Also recall that $\beta$ is given by

$$
\beta=\frac{\delta^{*}}{\tau_{0}} \frac{\mathrm{d} p_{1}}{\mathrm{~d} x}=-\frac{C_{1}[\Pi] S \delta_{c}}{U_{1}} \frac{\mathrm{d} U_{1}}{\mathrm{~d} x} .
$$

Substituting (B 10), (B 6) and (B 5) into (B 1) gives (4.8). Substituting (B 6) into (B 9) gives (4.9) and substituting (B 6) into (B 11) gives (4.10).

\section{Appendix C. Determining experimental values of $\Pi$ and $\delta_{c}$}

In the following, a systematic and self-consistent method for determining the parameters $\Pi$ and $\delta_{c}$ is proposed. First, it is assumed that the mean velocity profile is described well by the law of the wall and law of the wake given by (4.6). Once $U_{\tau}$ (i.e. $S$ ) has been found (e.g. by the Clauser chart method) the parameters can be determined based on a parametric curve fit of (4.6) to data. Various curve fit 
techniques can be used (e.g. see Jones et al. 2001, for a crude but commonly used technique for finding $\Pi$ ). A more accurate method is to use the experimental values of displacement thickness and momentum thickness in order to determine the parameters $\Pi$ and $\delta_{c}$.

From (B 2) and (B 3) the shape factor is,

$$
H=\frac{\delta^{*}}{\theta}=\frac{C_{1}[\Pi]}{C_{1}[\Pi]-C_{2}[\Pi] / S} .
$$

From (4.14) and (4.15) (C 1) is a quadratic in $\Pi$ and hence can be solved for $\Pi$ given the experimental values of $S$ and $H$. The appropriate root is

$$
\begin{aligned}
\Pi= & \frac{1}{2496 H}\left(-840 \kappa S-2140 H+840 H \kappa S+4\left(44100 \kappa^{2} S^{2}\right.\right. \\
& +28140 H \kappa S-88200 \kappa^{2} S^{2} H-92855 H^{2}-28140 H^{2} \kappa S \\
& \left.\left.+44100 H^{2} \kappa^{2} S^{2}\right)^{1 / 2}\right) .
\end{aligned}
$$

Having found $\Pi$ (B 3) can be solved for $\delta_{c}$.

\section{Appendix D. Some properties of the shear stress functions}

In the expression for shear stress (2.9)

$$
\frac{\tau}{\tau_{0}}=f_{1}[\eta, \Pi, S]+g_{1}[\eta, \Pi, S] \zeta+g_{2}[\eta, \Pi, S] \beta,
$$

the functions $f_{1}, g_{1}$ and $g_{2}$ are found to become independent of $S$ as $S \rightarrow \infty$ and are given by

$$
\begin{aligned}
& f_{1}[\eta, \Pi, \infty]=1+\frac{\left(\eta f-\int_{0}^{\eta} f \mathrm{~d} \eta\right)}{C_{1}[\Pi]}, \\
& g_{1}[\eta, \Pi, \infty]=\frac{\mathrm{d}}{\mathrm{d} \Pi} \int_{0}^{\eta} f \mathrm{~d} \eta-\frac{\mathrm{d} C_{1}[\Pi]}{\mathrm{d} \Pi} \frac{\left(\eta f-\int_{0}^{\eta} f \mathrm{~d} \eta\right)}{C_{1}[\Pi]}, \\
& g_{2}[\eta, \Pi, \infty]=\frac{2 \eta f}{C_{1}[\Pi]} .
\end{aligned}
$$

Here $f$ is the velocity defect, which using (2.5) can be expressed by

$$
f[\eta, \Pi]=\frac{U_{1}-U}{U_{\tau}}=-\frac{1}{\kappa} \log \eta+\frac{\Pi}{\kappa}\left(W_{c}[1, \Pi]-W_{c}[\eta, \Pi]\right)
$$

and which using formulation (4.6) is given by

$$
f[\eta, \Pi]=-\frac{1}{\kappa} \log \eta+\frac{1}{3 \kappa}\left(\eta^{3}-1\right)+\frac{\Pi}{\kappa}\left(2-6 \eta^{2}+4 \eta^{3}\right) .
$$

Two interesting results emerge by studying the functional forms of $f_{1}, g_{1}$ and $g_{2}$ at $S \rightarrow \infty$. First, as the Coles wake factor becomes very high, it can be shown that $g_{1}$ and $g_{2}$ become geometrically identical in shape and are related by

$$
-2 \kappa g_{1}[\eta, \infty, \infty]=g_{2}[\eta, \infty, \infty] .
$$

Secondly, by integrating the functions with respect to $\eta$ from 0 to 1 , we find that

$$
f_{1 i}[\Pi, \infty]=g_{2 i}[\Pi, \infty]
$$


and using (4.6) a surprisingly simple form emerges:

$$
f_{1 i}[\Pi, \infty]=\frac{6(1+2 \Pi)}{5(3+4 \Pi)} .
$$

\section{REFERENCES}

Bradshaw, P. \& Ferriss, D. 1965 The response of a retarded equilibrium boundary layer to the sudden removal of pressure gradient. Tech. Rep. 1145. NPL Aero. Report.

Chong, M. S., Soria, J., Perry, A. E., Chacin, J., Cantwell, B. J. \& Na, Y. 1998 Turbulence structures of wall-bounded shear flows found using DNS data. J. Fluid Mech. 357, 225-247.

Clauser, F. H. 1954 Turbulent boundary layers in adverse pressure gradients. J. Aero. Sci. 21, 91-108.

Clauser, F. H. 1956 The turbulent boundary layer. Adv. Mech. 4, 1-51.

Coles, D. E. 1956 The law of the wake in the turbulent boundary layer. J. Fluid Mech. 1, 191-226.

Coles, D. E. 1957 Remarks on the equilibrium turbulent boundary layer. J. Aero. Sci. 24, 459-506.

CoLES, D. E. 1962 The turbulent boundary layer in a compressible fluid. USAF The Rand Cooperation, Rep. R-403-PR, Appendix A.

Coles, D. E. \& Hirst, E. A. 1969 Compiled data. In Proc. Computation of Turbulent Boundary Layers, Vol. II. AFOSR-IFP-Stanford Conference 1968.

Dengel, G. \& Fernholz, H. H. 1990 An experimental investigation of an incompressible turbulent boundary layer in the vicinity of separation. J. Fluid Mech. 212, 615-636.

East, L. F., SAwYer, W. G. \& NASH, C. R. 1979 An investigation of the structure of equilibrium turbulent boundary layers. RAE Tech. Rep. 79040.

Green, J. E., Weeks, D. J. \& Brooman, J. W. F. 1973 Prediction of turbulent boundary layers and wakes in compressible flow by a lag-entrainment method. $A R C R$ and $M 3791$.

JONES, M. B. 1998 The structure of sink flow turbulent boundary layers. PhD thesis, University of Melbourne, Australia.

Jones, M. B., Marusic, I. \& Perry, A. E. 2001 Evolution and structure of sink flow turbulent boundary layers. J. Fluid Mech. 428, 1-27.

Lewkowicz, A. K. 1982 An improved universal wake function for turbulent boundary layers and some of its consequences. Z. Flugwiss. Weltraumforsch. 6, 261-266.

MARUSIC, I. 1991 The structure of zero- and adverse-pressure-gradient turbulent boundary layers. $\mathrm{PhD}$ thesis, The University of Melbourne, Australia.

Marusic, I. \& Perry, A. E. 1995 A wall-wake model for the turbulence structure of boundary layers. Part 2. Further experimental support. J. Fluid Mech. 298, 389-407.

PERry, A. E. 1992 A new look at some closure problems of turbulent boundary layers. GALCIT Rep. FM-92-4. California Institute of Technology.

Perry, A. E. \& Fairlie, B. D. 1975 A study of turbulent boundary layer separation and reattachment. J. Fluid Mech. 69, 657-672.

Perry, A. E., LI, J. D. \& Marusic, I. 1991 Towards a closure scheme for turbulent boundary layers using the attached eddy hypothesis. Phil. Trans. R. Soc. Lond. A 336, 67-79.

Perry, A. E. \& Marusic, I. 1995 A wall-wake model for the turbulence structure of boundary layers. Part 1. Extension of the attached eddy hypothesis. J. Fluid Mech. 298, 361-388.

Perry, A. E., Marusic, I. \& Jones, M. B. 1998 New evolution equations for turbulent boundary layers in arbitrary pressure gradients. Sadhana, Indian Acad. Sci. 23 (5),(6), 443-457.

Perry, A. E., Marusic, I. \& Li, J. D. 1994 Wall turbulence closure based on classical similarity laws and the attached eddy hypothesis. Phys. Fluids 2, 1024-1035.

Rotta, J. C. 1962 Turbulent boundary layers in incompressible flow. Prog. Aero. Sci. 2, 1-219.

Samuel, A. E. \& Joubert, P. N. 1974 A boundary layer developing in an increasingly adverse pressure gradient. J. Fluid Mech. 66, 481-505.

SkÅre, P. E. \& KrogStad, P.-Å. 1994 A turbulent boundary layer near separation. J. Fluid Mech. 272, 319-348.

Townsend, A. A. 1956 The Structure of Turbulent Shear Flow. Cambridge University Press. Townsend, A. A. 1976 The Structure of Turbulent Shear Flow, vol. 2. Cambridge University Press. White, F. M. 1974 Viscous Fluid Flow. McGraw-Hill. 


\section{University Library}

\section{- M M I N E R VA A gateway to Melbourne's research publications}

Minerva Access is the Institutional Repository of The University of Melbourne

Author/s:

Perry, A. E.;Marusic, I.;Jones, M. B.

Title:

On the streamwise evolution of turbulent boundary layers in arbitrary pressure gradients

Date:

2002

Citation:

Perry, A. E., Marusic, I. \& Jones, M. B. (2002). On the streamwise evolution of turbulent boundary layers in arbitrary pressure gradients. Journal of Fluid Mechanics, 461, 61-91.

Publication Status:

Published

Persistent Link:

http://hdl.handle.net/11343/34805 